\title{
Holographic stress tensor at finite coupling
}

\author{
Kallol Sen and Aninda Sinha \\ Centre for High Energy Physics, Indian Institute of Science, \\ C.V. Raman Avenue, Bangalore 560012, India \\ E-mail: kallol@cts.iisc.ernet.in, asinha@cts.iisc.ernet.in
}

ABSTRACT: We calculate one, two and three point functions of the holographic stress tensor for any bulk Lagrangian of the form $\mathcal{L}\left(g^{a b}, R_{a b c d}, \nabla_{e} R_{a b c d}\right)$. Using the first law of entanglement, a simple method has recently been proposed to compute the holographic stress tensor arising from a higher derivative gravity dual. The stress tensor is proportional to a dimension dependent factor which depends on the higher derivative couplings. In this paper, we identify this proportionality constant with a B-type trace anomaly in even dimensions for any bulk Lagrangian of the above form. This in turn relates to $\mathcal{C}_{T}$, the coefficient appearing in the two point function of stress tensors. We use a background field method to compute the two and three point function of stress tensors for any bulk Lagrangian of the above form in arbitrary dimensions. As an application we consider general situations where $\eta / s$ for holographic plasmas is less than the KSS bound.

KEYWORDS: Gauge-gravity correspondence, AdS-CFT Correspondence

ARXiv EPRINT: 1405.7862 


\section{Contents}

1 Introduction 2

2 Stress tensor from first law of entanglement $\quad 6$

2.1 For $\mathcal{L}\left(g^{a b}, R_{\text {cdef }}\right) \quad 8$

2.2 For $\mathcal{L}\left(g^{a b}, R_{c d e f}, \nabla_{a} R_{b c d e}\right) \quad 9$

3 Holographic trace anomalies $\quad 10$

3.1 For $\mathcal{L}\left(g^{a b}, R_{\text {cdef }}\right) \quad 10$

$\begin{array}{lll}3.1 .1 \mathrm{~d}=2 & 11\end{array}$

$\begin{array}{lll}3.1 .2 \mathrm{~d}=4 & 12\end{array}$

$\begin{array}{ll}3.1 .3 \mathrm{~d}=6 & 12\end{array}$

$3.2 \quad$ For $\mathcal{L}\left(g^{a b}, R_{\text {cdef }}, \nabla_{a} R_{b c d e}\right) \quad 13$

$\begin{array}{lll}3.2 .1 \mathrm{~d}=4 & 14\end{array}$

$\begin{array}{ll}3.2 .2 \mathrm{~d}=6 & 14\end{array}$

4 Holographic two point function for higher derivative theories in arbitrary dimensions

$\begin{array}{llr}5 & \text { Three point functions } & \mathbf{1 7}\end{array}$

$\begin{array}{lll}5.1 \mathcal{L}\left(g^{a b}, R_{\text {cdef }}\right) & 18\end{array}$

$\begin{array}{lll}5.2 \mathcal{L}\left(g^{a b}, R_{\text {cdef }}, \nabla_{a} R_{\text {bcde }}\right) & 19\end{array}$

6 Application: $\eta / s$ for higher derivative theories $\quad 19$

$\begin{array}{lll}7 & \text { Discussion } & 23\end{array}$

A The Lagrangian in terms of [17] 24

B Details of calculation for section (2.2) 26

C Holographic stress tensor involving $\nabla R$ terms $\quad 27$

$\begin{array}{lll}\text { C. } 1 \mathrm{~d}=4 & 27\end{array}$

$\begin{array}{lll}\text { C. } 2 \mathrm{~d}=6 & 28\end{array}$

$\mathrm{D}\left\langle\boldsymbol{T}_{\mu \nu}(x) T_{\rho \sigma}(0)\right\rangle$ in even dimensions $\quad 28$

$\begin{array}{ll}\text { D. } \mathrm{d}=2 & 29\end{array}$

D. $2 \mathrm{~d}=4 \quad 30$

D. $3 \mathrm{~d}=6 \quad 31$

E $\quad \eta / s$ for general $R^{2}$ theories $\quad 32$ 


\section{Introduction}

Holographic methods have proved to be enormously useful to gain intuition about certain physical questions at strong coupling [1-3]. However, in most applications, attention has focused on cases where the bulk gravitational dual is two derivative. In the AdS/CFT dictionary, this corresponds to infinite 't Hooft coupling and an infinite number of colours. In order to make possible contact with the real world, one needs to consider effects due to finite 't Hooft coupling and a finite number of colours. In the best studied example corresponding to the $\mathcal{N}=4 \mathrm{SU}(\mathrm{N})$ supersymmetric Yang-Mills with the gravitational dual being type IIB superstring theory on $\mathrm{AdS}_{5} \times \mathrm{S}^{5}$, finite coupling corrections correspond to specific higher derivative corrections in the low energy effective action of string theory. In addition to these corrections, there are also non-local contributions, for example from graviton loops. One essential step to take into account the contributions at finite coupling, is to be able to compute the holographic stress tensor and its correlation functions for an arbitrary higher derivative theory of gravity.

Calculating the holographic stress tensor itself from first principles $[4,5]$ appears to be prohibitively difficult except for certain cases where the generalized Gibbons-Hawking term and the counterterms are known. Since the generalized Gibbons-Hawking term is not known for an arbitrary higher curvature theory, this stymies any progress using conventional approaches - only some sporadic results for specific examples are known in the literature [6-16]. Recently, a way around has been found using the first law of entanglement pertaining to spherical entangling surfaces [17]. The way this works is as follows: the first law of entanglement states that $[18,19]$

$$
\Delta S=\Delta H,
$$

where for two density matrices $\rho, \sigma$ with $\sigma \equiv e^{-H} / \operatorname{tr} e^{-H}$ being the reduced density matrix for a spherical entangling surface in a CFT with $H$ being the modular hamiltonian, $\Delta H=$ $\langle H\rangle_{\rho}-\langle H\rangle_{\sigma}$ and $\Delta S=S(\rho)-S(\sigma)$ with $S(\rho)=-\operatorname{tr} \rho \log \rho$ being the von Neumann entropy for $\rho$ and is the entanglement entropy for a reduced density matrix $\rho$. The equality arises at linear order in perturbation, meaning that $\rho, \sigma$ belong to some family of density matrices $\hat{\rho}$ parametrized by some perturbation parameter $\lambda$ such that $\sigma=\hat{\rho}(\lambda=0), \rho=$ $\hat{\rho}(\lambda)$ and we are interested only in linear order in $\lambda$. At nonlinear order in $\lambda$ we get an inequality which corresponds to the positivity of relative entropy, leading to $\Delta H>\Delta S$. The expression for $H$ (which will be given below) involves the time-time component of the field theory stress tensor. In holography, for spherical entangling surface, the entanglement entropy for the vacuum state across the sphere $S^{d-2}$ gets mapped to the thermal entropy on $R \times H^{d-1}$. Using the gravitational dual, the thermal entropy is computed using the Wald entropy which is known for an arbitrary higher derivative theory of gravity. For bifurcate Killing horizons, there is a theorem due to Iyer and Wald [20] which states that linearized perturbations satisfy the first law of thermodynamics which translated to our case means that eq. (1.1) would be applicable with linearized perturbations in the Wald formula. Thus the l.h.s. of eq. (1.1) can be computed using the linearization of the Wald formula. The r.h.s. of eq. (1.1) has the perturbation of the time-time component of the field 
theory stress tensor which now can be determined. In order to be able to do the integral, one approximates the entangling surface radius $R$ to be small. Since the only dimensionless parameter is $R^{d}\left\langle T_{\mu \nu}\right\rangle$, the perturbative expansion can be done either by treating $R$ to be small or by treating $\left\langle T_{\mu \nu}\right\rangle$ to be small. Thus although the expression for the stress tensor obtained using the above logic pertains to an excited state that is a small perturbation from the vacuum state, the expression should hold for any $\left\langle T_{\mu \nu}\right\rangle$. Since this can be done for an arbitrary higher derivative theory, we thus know how to compute the holographic stress tensor for such a bulk dual.

The result of this calculation is a very compact expression for the stress tensor in terms of certain parameters appearing in the linearized expression of the Wald formula. In particular, if one ignores covariant derivatives of the curvature tensor, the result can be worked out quite simply. Writing the linearized Wald functional as

$$
\delta E_{R}^{a b c d}=-c_{2} g^{\langle a b} g^{c d\rangle} h-c_{3} h^{\langle a b} g^{c d\rangle}+c_{4} g^{\langle a b} g^{c d\rangle} \mathcal{R}+c_{5} \mathcal{R}^{\langle a b} g^{c d\rangle}+c_{6} \mathcal{R}^{a b c d},
$$

where

$$
\delta g^{a b}=-h^{a b}, \quad \delta R^{a b c d}=\mathcal{R}^{a b c d}, \quad h=g_{c d} h^{c d},
$$

one finds that ${ }^{1,2}$

$$
\left\langle T_{\mu \nu}\right\rangle=d \tilde{L}^{d-3}\left[c_{1}+2(d-2) c_{6}\right] h_{\mu \nu}^{(d)},
$$

where $h_{\mu \nu}^{(d)}$ appears in the Fefferman-Graham expansion of the asymptotic AdS metric as

$$
d s^{2}=\tilde{L}^{2} \frac{d z^{2}}{z^{2}}+\frac{1}{z^{2}}\left(g_{\mu \nu}^{(0)}+z^{2} g_{\mu \nu}^{(2)}+\cdots z^{d} h_{\mu \nu}^{(d)}+\cdots\right) d x^{\mu} d x^{\nu} .
$$

$\tilde{L}$ is the AdS radius. This begs the question: what is this simple proportionality constant depending on $c_{i}$ 's in (1.4)? Since the linearized Wald functional was involved in the derivation of this simple form, with hindsight we can anticipate that there are simplifications waiting to happen if we consider rewriting the Lagrangian as a background field expansion around a suitable background. Recently this background field method has been used to find simple expressions for trace anomalies in even dimensions [21]. We will make a simple modification to this method so that the anomaly calculation can be carried out easily using Mathematica. Let us now explain why this method is useful in correlating with the results above as well as calculating higher point correlation functions. Given a Lagrangian $\mathcal{L}\left(g^{a b}, R_{b c d e}, \nabla_{f} R_{b c d e}, \cdots\right)$, we are going to treat $g_{a b}$ and $R_{a b c d}$ as independent variables. We are going to expand $R_{a b c d}$ around $\bar{R}_{a b c d}=-\frac{1}{\tilde{L}^{2}}\left(g_{a c} g_{b d}-g_{a d} g_{b c}\right)$ where $g_{a b}$ in this expression is the full metric. Raising and lowering indices and the covariant derivative is done using the full metric. Define $\Delta R_{a b c d}=R_{a b c d}-\bar{R}_{a b c d}$. Then on the AdS background $\left(g_{\mu \nu}^{(0)}=\eta_{\mu \nu}\right)$ this quantity is zero. Further if we linearize this then in the transverse traceless gauge, it can be easily checked that $\left(\Delta R_{a b}\right)^{L}=(\Delta R)^{L}=0$. This is the reason why the expressions we will compute for the stress tensor correlation functions will take on simple

\footnotetext{
${ }^{1}$ Note that we are considering field theory in flat space.

${ }^{2}$ The normalization of $\Delta H$ is fixed by the definition of modular Hamiltonian in (1.1). On the r.h.s. the normalization of $\Delta S$ is fixed by holography where we demand that the definition of $S$ gives the correct universal terms. This resolves any ambiguity in the definition of $\left\langle T_{\mu \nu}\right\rangle$ in (1.4) by using $h_{\mu \nu}^{(d)}$ in (1.5).
} 
forms. Let us start with $\mathcal{L}\left(g^{a b}, R_{b c d e}\right)$, i.e., no covariant derivatives (we will set the AdS radius $\tilde{L}=1$ from hereon and will reinstate it when needed). The Lagrangian after doing the background field expansion takes the form

$$
\mathcal{L}=\left(c_{0}+c_{1} \Delta R+\frac{c_{4}}{2} \Delta R^{2}+\frac{c_{5}}{2} \Delta R^{a b} \Delta R_{a b}+\frac{c_{6}}{2} \Delta R^{a b c d} \Delta R_{a b c d}+\sum_{i=1}^{8} \tilde{c}_{i} \Delta \mathcal{K}_{i}+\cdots\right)
$$

where $c_{0}=-2 d c_{1}$ and $\Delta \mathcal{K}_{i}=\left.\mathcal{K}_{i}\right|_{R \rightarrow(R-\bar{R})}$. Note that we are not treating $c_{i}$ 's perturbatively. This Lagrangian can be shown to lead to (1.2). The basis for the third order terms is given by

$$
\begin{array}{r}
\mathcal{K}_{i}=\left(R^{3}, R_{b}^{a} R_{c}^{b} R_{a}^{c}, R R^{a b} R_{a b}, R R^{a b c d} R_{a b c d}, R^{a b} R^{c d} R_{a c b d}, R_{a b} R^{a c d e} R_{c d e}^{b},\right. \\
\left.R_{a b c d} R^{a b e f} R_{\text {ef }}^{c d}, R_{a b c d} R^{a e f c} R_{e f}^{b}{ }^{d}\right) .
\end{array}
$$

We are not using an explicit overall factor of $1 / 2 \ell_{p}^{d-1}$ with the action since all the coefficients in the action are assumed to implicitly have the factor. In order to compute $n$-point functions we expand the bulk action up to $n$ 'th order in the perturbation. However, since $\Delta R_{a b c d}^{\mathrm{AdS}}=0$, this means that we only need to retain up to $O\left((\Delta R)^{n}\right)$ terms in the Lagrangian. Thus, the background field expanded Lagrangian is an expansion in terms of the correlation functions of the stress tensor. Further simplifications happen. Consider $(\Delta R)^{2}$ or $\left(\Delta R_{a b}\right)^{2}$. Since the linearized $\Delta R$ and $\Delta R_{a b}$ both vanish, these terms can only contribute to four-point functions onwards. Thus we do not expect $c_{4}$ or $c_{5}$ in eq. (1.6) above to enter the one, two or three point functions. This is consistent with the absence of these coefficients in eq. (1.4). Moreover, this conclusion does not change on including covariant derivatives.

Let us now summarize our findings for the correlation functions that follow from the Lagrangian in eq. (1.6). The stress tensor two point function takes the form

$$
\left\langle T_{a b}(x) T_{c d}\left(x^{\prime}\right)\right\rangle=\frac{\mathcal{C}_{T}}{\left|x-x^{\prime}\right|^{2 d}} \mathcal{I}_{a b, c d}\left(x-x^{\prime}\right),
$$

where

$$
\mathcal{I}_{a b, c d}(x)=\frac{1}{2}\left[I_{a b}(x) I_{c d}(x)+I_{a d}(x) I_{b c}(x)\right]-\frac{1}{d} \eta_{a b} \eta_{c d},
$$

and

$$
I_{a b}(x)=\eta_{a b}-2 \frac{x_{a} x_{b}}{x^{2}} .
$$

The coefficient $\mathcal{C}_{T}$ from the $d+1$ dimensional bulk Lagrangian works out to be

$$
\mathcal{C}_{T}=f_{d} \tilde{L}^{d-1}\left[c_{1}+2(d-2) c_{6}\right],
$$

where $\tilde{L}$ is the AdS radius and $f_{d}$ is constant $d$ dependent factor given by [22]

$$
f_{d}=2 \frac{d+1}{d-1} \frac{\Gamma[d+1]}{\pi^{d / 2} \Gamma[d / 2]} .
$$

Thus the holographic stress tensor in eq.(1.4) can be written as

$$
\left\langle T_{\mu \nu}\right\rangle=\frac{d}{\tilde{L}^{2} f_{d}} \mathcal{C}_{T} h_{\mu \nu}^{(d)} .
$$


Further for even dimensional CFTs the coefficient $\mathcal{C}_{T}$ is related to a B-type anomaly coefficient as we will show. In particular, the A-type Euler anomaly coefficient is simply proportional to $c_{1}$ while the B-type anomaly coefficient (conventionally called $c$ in $4 d$ and $B_{3}$ in $6 d$ ) is proportional to $C_{T}$.

We use the method of background field expansion to calculate the three point functions of stress tensor. Following the simple method devised in $[23,24]$ and used in $[25,26]$ we perform the calculation of the three point function in a shockwave background and obtain information about the three point function from the energy flux given by (these results are for $d \geq 4$, for $d=3$, the term proportional to $t_{2}$ is absent),

$$
\langle\epsilon(\mathbf{n})\rangle=\frac{E}{4 \pi}\left[1+t_{2}\left(\frac{\epsilon_{i j}^{*} \epsilon_{i k} n^{j} n^{k}}{\epsilon_{i j}^{*} \epsilon_{i j}}-\frac{1}{d-1}\right)+t_{4}\left(\frac{\left|\epsilon_{i j} n^{j} n^{k}\right|^{2}}{\epsilon_{i j}^{*} \epsilon_{i j}}-\frac{2}{d^{2}-1}\right)\right],
$$

where,

$$
\begin{aligned}
t_{2} & =\frac{d(d-1)}{c_{1}+2(d-2) c_{6}}\left[2 c_{6}-12(3 d+4) \tilde{c}_{7}+3(7 d+4) \tilde{c}_{8}\right], \\
t_{4} & =\frac{6 d\left(d^{2}-1\right)(d+2)}{c_{1}+2(d-2) c_{6}}\left(2 \tilde{c}_{7}-\tilde{c}_{8}\right) .
\end{aligned}
$$

$\mathbf{n}$ is the unit normal in the direction in which energy flux is measured and $t_{2}$ and $t_{4}$ are determined holographically. The coefficients $t_{2}, t_{4}$ and $\mathcal{C}_{T}$ are related to the three independent coefficients appearing in the three point functions $[27,28] .{ }^{3}$ Notice that for $d=4$, the $\tilde{c}_{7}, \tilde{c}_{8}$ dependence in $t_{2}$ and $t_{4}$ are packaged in the same way, namely as $2 \tilde{c}_{7}-\tilde{c}_{8}$. This is indicative of the fact that $c, a, t_{2}, t_{4}$ satisfy the relation $(c-a) / c=t_{2} / 6+4 t_{4} / 45$. This relation enables one to extract the $4 \mathrm{~d}$ Euler anomaly $a$ from the knowledge of two and three point functions. In six (and higher) dimensions, there is no such relation (in fact not even for a linear combination of the A-anomaly and the B-anomaly coefficients) indicating the fact that a similar relation involving the Euler anomaly coefficient will also involve higher point correlation functions.

We can easily extend the above results to the $\mathcal{L}\left(g^{a b}, R_{b c d e}, \nabla_{f} R_{b c d e}\right)$ case, i.e., to the situation where there are at most two covariant derivatives of the curvature tensor in the action. First notice that since the linearized $\Delta R_{a b}$ and $\Delta R$ both vanish, only terms like $\nabla_{e} \Delta R_{a b c d} \nabla^{e} \Delta R^{a b c d}$ will contribute to the two and three point functions while only terms like $\Delta R_{\ldots} \nabla \Delta R_{\ldots} \nabla \Delta R_{\ldots}$ will contribute to the three point functions. Further, we will show that using the Bianchi identities and integration by parts [29], the $\nabla_{e} \Delta R_{a b c d} \nabla^{e} \Delta R^{a b c d}$ terms can be rewritten in terms of $\left(\Delta R_{\ldots}\right)^{3}$ and $\nabla_{a} \Delta R_{b c} \nabla^{a} \Delta R^{b c}, \nabla_{a} \Delta R \nabla^{a} \Delta R$. Since the

${ }^{3}$ The relation between $t_{2} t_{4}$ and $\mathcal{C}_{T}$ and the CFT coefficients $\mathcal{A}, \mathcal{B}$ and $\mathcal{C}$ are

$$
\begin{aligned}
\mathcal{C}_{T} & =\frac{\Omega}{2 d(d+2)}[(d-1)(d+2) \mathcal{A}-2 \mathcal{B}-4(d+1) \mathcal{C}], \\
t_{2} & =\frac{2(d+1)}{d} \frac{(d-2)(d+2)(d+1) \mathcal{A}+3 d^{2} \mathcal{B}-4 d(2 d+1) \mathcal{C}}{(d-1)(d+2) \mathcal{A}-2 \mathcal{B}-4(d+1) \mathcal{C}}, \\
t_{4} & =-\frac{d+1}{d} \frac{(d+2)\left(2 d^{2}-3 d-3\right) \mathcal{A}+2 d^{2}(d+2) \mathcal{B}-4 d(d+1)(d+2) \mathcal{C}}{(d-1)(d+2) \mathcal{A}-2 \mathcal{B}-4(d+1) \mathcal{C}} .
\end{aligned}
$$


last two terms do not contribute to two or three point functions, the result for the two point functions will involve a redefined $c_{6}$. We will explicitly show that the result for the three point functions also follows a similar simple trend.

As an application for our methods we will compute the ratio of shear viscosity $(\eta)$ to entropy density $(s)$ for a general four derivative bulk dual, without assuming the coupling constants to be small (for earlier related work see [30-40]). Then following [25, 26], we will demand that $-3 \leq t_{2} \leq 3$ as well as $\mathcal{C}_{T}>0, s>0$. These constraints were sufficient in the Gauss-Bonnet case $[23,41,42]$ to lead to $\eta / s \geq \frac{16}{25} \frac{1}{4 \pi} \approx 0.64 \frac{1}{4 \pi}$. We will find that in the general four derivative case, we can tune the couplings so that these conditions are satisfied but $\eta / s$ is arbitrarily small. This is of course due to the fact that the underlying theory has non-unitary modes. We will also show that for the Weyl-squared theory, the above constraints lead to $\eta / s \geq \frac{12-3 \sqrt{2}}{14} \frac{1}{4 \pi} \approx 0.55 \frac{1}{4 \pi}$ while including Weyl-cubed terms, the same constraints lead to $\eta / s \geq 0.17 \frac{1}{4 \pi}$. Both these theories will have non-unitary modes supported near the horizon. It is interesting to note that there is still a bound on the ratio in such theories.

The rest of the paper is organised as follows. In section (2.1) we give a brief review of the calculations of [17]. In section (3.1) we calculate the holographic trace anomalies by considering the background field expanded Lagrangian and also how various coefficients of the Lagrangian in [17] are related to the Lagrangian we are considering. We then compute the trace anomalies in $d=2,4,6$ and show that the B-type anomalies are the coefficients in the expression for the holographic stress tensor. In section (3.2) we extend the analysis to Lagrangians containing covariant derivatives on the Riemann tensors and show how the anomaly coefficients get modified. More specifically we show that $c_{6}$ in the B-type anomaly coefficients can be replaced by an effective $c_{6}^{\prime}$ in presence of the $\nabla R$ terms in the Lagrangian. In section (4) and section (5) we extend the analysis to calculating the holographic two and three point functions of the stress tensor. We show that the coefficient in the holographic one point function of the stress tensor is related to the coefficient of the holographic two point functions of the stress tensor for arbitrary dimensions. Section (6) presents one application of the method of background field expansion in the calculation $\eta / s$. We present the calculations for Weyl-squared, Weyl cubed and general $R^{2}$ gravity (appendix (E)). We also show that the bounds for $\eta / s$ for these theories pertaining to the physical constraints satisfied by the three point functions are much smaller that the KSS bound [43-45]. We end with a discussion about open problems in section (7).

\section{Stress tensor from first law of entanglement}

In this section we review the derivation of the holographic stress tensor from the first law of entanglement [18, 19] for (1.6) following [17]. The modular hamiltonian for a spherical entangling region of radius $R$ and centered around $x=x_{0}$, is given by

$$
H_{A}=2 \pi \int_{A\left(R, x_{0}\right)} d^{d-1} x \frac{R^{2}-\left|\mathbf{x}-\mathbf{x}_{\mathbf{0}}\right|^{2}}{2 R}\left\langle T_{t t}\right\rangle
$$


and for any perturbation around the CFT vacuum we have

$$
\Delta H_{A}=2 \pi \int_{A\left(R, x_{0}\right)} d^{d-1} x \frac{R^{2}-\left|\mathbf{x}-\mathbf{x}_{\mathbf{0}}\right|^{2}}{2 R} \delta T_{t t} .
$$

As mentioned in [17], the entanglement entropy of the spherical entangling region in the vacuum CFT is equal to the entropy of a thermal CFT on a hyperbolic cylinder with the temperature set by the length scale of the hyperbolic spacetime. From the holographic side the thermal entropy is given by the entropy of the hyperbolic black hole, which for any classical higher derivative theory of gravity is evaluated using the Wald formula [20]

$$
S^{\mathrm{Wald}}=-2 \pi \int_{\mathcal{H}} d^{n} \sigma \sqrt{h} \frac{\delta \mathcal{L}}{\delta R_{c d}^{a b}} n^{a b} n_{c d}
$$

where $\mathcal{L}$ is given in (1.6) and $n^{a b}$ is the unit binormal to the bifurcate Killing horizon $\mathcal{H}$. In general the Wald entropy functional differs from the enanglement entropy functional by squares of the extrinsic curvature [46-53] but for the spherical entangling region these terms vanish and $S^{\text {Wald }}=S_{E E}$ at the linear order in perturbations [17]. Further, the perturbations of the vacuum CFT imply perturbations of the thermal CFT since the perturbations of the vacuum AdS imply perturbations of each of the AdS-Rindler wedges for the thermal state. ${ }^{4}$

Before proceeding we will specify the notations and conventions. Throughout we set the AdS radius. ${ }^{5} \tilde{L}=1$ except where we explicitly restore it on dimensional grounds. $R$ is the radius of the entangling ball. In terms of the Poincaré coordinates, AdS spacetime is given by,

$$
d s^{2}=\frac{d z^{2}+\eta_{\mu \nu} d x^{\mu} d x^{\nu}}{z^{2}} .
$$

The spherical entangling region $A$ in the vacuum CFT is associated with the hemispherical region $\tilde{A}$ in the black hole background given by $\tilde{A}=\left\{t=t_{0}, \quad\left(x^{i}-x_{0}^{i}\right)^{2}+z^{2}=R^{2}\right\}$ in Poincaré coordinates. These two different regions have the same boundary $\partial A$ in the boundary CFT. Thus $S_{E E}$ is equal to $S^{\text {Wald }}$ evaluated on $\tilde{A}$. Similarly the perturbation $\triangle S_{E E}$ of the vacuum CFT is equal to $\delta S^{\text {Wald }}$. For holographic CFTs the gravitational version of $\delta S_{A}=\delta E_{A}$ is given by $\delta S^{\text {grav }}=\delta E^{\text {grav }}=\delta S^{\text {Wald }}$ and can be used to relate the $\left\langle T_{\mu \nu}\right\rangle$ to the asymptotic form of the metric in the holographic side. In the limit of $R \rightarrow 0$, $\delta\left\langle T_{t t}\left(t_{0}, \mathbf{x}\right)\right\rangle$ can be replaced by its central value $\delta\left\langle T_{t t}\left(x_{0}\right)\right\rangle$ and we have using $\delta E_{A}=\delta S_{A}$,

$$
\delta\left\langle T_{t t}\left(x_{0}\right)\right\rangle=\frac{d^{2}-1}{2 \pi \Omega_{d-2}} \lim _{R \rightarrow 0}\left(\frac{1}{R^{d}} \delta S_{A}^{\text {Wald }}\right)
$$

and repeating for arbitrary Lorentz frames we have

$$
u^{\mu} u^{\nu} \delta\left\langle T_{\mu \nu}\left(x_{0}\right)\right\rangle=\frac{d^{2}-1}{2 \pi \Omega_{d-2}} \lim _{R \rightarrow 0}\left(\frac{1}{R^{d}} \delta S_{A}^{\mathrm{Wald}}\right) .
$$

\footnotetext{
${ }^{4}$ This assumption is only valid at the leading order in perturbations. In the next order the hyperbolic horizon changes due to the perturbations and we do not have the AdS-Rindler patch.

${ }^{5}$ Note that $L$ is the length associated with the cosmological constant.
} 
The variation of the Wald entropy around the hyperbolic black hole background for an arbitrary higher derivative theory of gravity is given by

$$
\delta S^{\mathrm{Wald}}=\delta\left(-2 \pi \int_{\tilde{A}} E_{R}^{a b c d} \epsilon_{a b} n_{c d}\right)
$$

where $E^{a b c d}$ is the Wald functional of the curvatures and their covariant derivatives, $\epsilon_{a b}$ is the volume element and $n_{c d}=n_{c}^{1} n_{d}^{2}-n_{d}^{1} n_{c}^{2}$ is the binormal to the bifurcation surface $\bar{B}$ respectively.

\section{$2.1 \quad$ For $\mathcal{L}\left(g^{a b}, R_{c d e f}\right)$}

Evaluated on an AdS background where $R_{a b c d}=-\left(g_{a c} g_{b d}-g_{a d} g_{b c}\right)$, it can be shown (see appendix A) that the Wald functional and its linear variation for (1.6) (without covariant derivatives of curvature terms in the action) takes the simple form

$$
E_{R}^{a b c d}=c_{1} g^{\langle a b} g^{c d\rangle}
$$

and,

$$
\delta E_{R}^{a b c d}=-c_{2} g^{\langle a b} g^{c d\rangle} h-c_{3} h^{\langle a b} g^{c d\rangle}+c_{4} g^{\langle a b} g^{c d\rangle} \mathcal{R}+c_{5} \mathcal{R}^{\langle a b} g^{c d\rangle}+c_{6} \mathcal{R}^{a b c d},
$$

where all the coefficients are not independent but related by [17]

$$
c_{2}=-2 d c_{4}-c_{5}, \quad c_{3}=2 c_{1}-(d-1) c_{5}-4 c_{6},
$$

and $\langle$,$\rangle implies that it has been properly (anti)symmetrized to have the properties of the$ Riemann tensor. The linearized Reimann tensor is given by

$$
\mathcal{R}_{a b c d}=\frac{1}{2}\left(\nabla_{c} \nabla_{b} h_{a d}-\nabla_{d} \nabla_{b} h_{a c}+\nabla_{d} \nabla_{a} h_{b c}-\nabla_{c} \nabla_{a} h_{b d}\right)+\frac{1}{2}\left(R_{a e c d} h_{b}^{e}+R_{b c d}^{e} h_{a e}\right) .
$$

When $\delta T_{\mu \nu}$ is small and $R \rightarrow 0$, the scaling analysis in [17] shows that at the leading order we can neglect all the derivatives $\partial_{\mu \neq z}$ in comparison to $\partial_{z}$. Near the boundary, the metric perturbations can be written as

$$
h_{\mu \nu}\left(z, x^{\lambda}\right)=z^{\Delta-2} h_{\mu \nu}\left(x^{\lambda}\right)+\ldots
$$

Using (2.11) the relevant components of the linearized Wald functional in (1.2) take the form

$$
\delta E_{R}^{(1) \mu z \nu z}=A h^{\mu \nu} g^{z z}+B h g^{\mu \nu} g^{z z}, \delta E_{R}^{(1) \mu \nu \rho \sigma}=C h g^{\langle\mu \nu} g^{\rho \sigma\rangle}+D h^{\langle\mu \nu} g^{\rho \sigma\rangle},
$$

where the coefficients $A, B, C, D$ are functions of the coefficients ${ }^{6}$ in (1.6). Substituting (2.13) and (2.8) into (2.7), we get,

$$
\delta S^{\mathrm{Wald}}=\frac{4 \pi \tilde{L}^{d-3}}{R} \int_{A} \frac{d^{d-1} x}{z^{d-2}}\left(A_{1} h_{t t}+A_{2} \eta^{\mu \nu} h_{\mu \nu}\right)
$$

\footnotetext{
${ }^{6}$ The explicit dependences of $A, B, C$ and $D$ on the coefficients $c_{1} \ldots c_{6}$ are given in footnote 20 of [17].
} 
After putting $\Delta=d$ in order to get a finite answer [17] we find

$$
\begin{aligned}
& A_{1}=2\left(A-\frac{D}{4}\right)(d-2) R^{2}+\left[\frac{c_{1}}{2}-\frac{D}{2}(d-2)+2 A(d-1)\right]\left(\frac{|x|^{2}}{d-1}-R^{2}\right), \\
& A_{2}=\left(\frac{c_{1}}{2}+2 B\right) R^{2}+\left[\frac{c_{1}}{2}+\frac{D}{2}+(C-2 B)(d-1)\right] \frac{|x|^{2}}{d-1}
\end{aligned}
$$

Performing the integral in (2.14) and using (2.5) we have,

$$
\delta T_{t t}^{\mathrm{grav}}=\alpha h_{t t}^{(d)}+\beta \eta_{t t} h_{\mu}^{(d) \mu},
$$

where the coefficients are given as

$$
\begin{aligned}
\alpha & =d\left(-c_{1}+c_{3}+(d-1) c_{5}+2 d c_{6}\right), \\
\beta & =\left[-(d+2) c_{1}+2(d+1) c_{2}+c_{3}+2 d(d+1) c_{4}+(d+1) c_{5}-2(d-2) c_{6}\right],
\end{aligned}
$$

and $h_{\mu \nu}^{(d)}$ has no $z$ dependence. These can be generalized to an arbitrary Lorentz frame and combined with the tracelessness and conservation equations $h_{\mu}^{(d) \mu}=0, \partial_{\mu} h^{(d) \mu \nu}=0$ we have (1.4) as

$$
\delta T_{\mu \nu}^{\text {grav }}=d \tilde{L}^{d-3}\left[c_{1}+2(d-2) c_{6}\right] h_{\mu \nu}^{(d)} .
$$

\subsection{For $\mathcal{L}\left(g^{a b}, R_{c d e f}, \nabla_{a} R_{b c d e}\right)$}

The above analysis can be extended to actions containing covariant derivatives on the Riemann tensors. The most general term containing arbitrary covariant derivatives on the curvature tensors is deferred for futute work; we consider here $\mathcal{L}\left(g^{a b}, R_{c d e f}, \nabla_{g} R_{c d e f}\right)$. The background field expansion of the action at $O\left((\Delta R)^{2}\right)$ is given by

$$
S_{\nabla R}=\int d^{d+1} x \sqrt{g} Z^{\text {efabcdmnrs }} \nabla_{e} \Delta R_{a b c d} \nabla_{f} \Delta R_{m n r s} .
$$

It can be shown (see appendix B) that the above action can be written as

$$
S=\int d^{d+1} x \sqrt{g}\left[d_{1} \Delta R^{a b c d} \nabla^{2} \Delta R_{a b c d}+d_{2} \Delta R^{a b} \nabla^{2} \Delta R_{a b}+d_{3} \Delta R \nabla^{2} \Delta R\right] .
$$

Since $\nabla_{a} g_{b c}=0$, we can write

$$
S=\int d^{d+1} x \sqrt{g}\left[d_{1} \Delta R^{a b c d} \nabla^{2} R_{a b c d}+d_{2} \Delta R^{a b} \nabla^{2} R_{a b}+d_{3} \Delta R \nabla^{2} R\right] .
$$

At the linear order in fluctuations using (2.11)

$$
\begin{aligned}
\mathcal{R}_{a b}^{L} & =\frac{1}{2} \nabla^{c} \nabla_{a} h_{b c}+\frac{1}{2} \nabla_{b} \nabla^{d} h_{a d}-\frac{1}{2} \nabla^{2} h_{a b}-\frac{1}{2} \nabla_{a} \nabla_{b} h+\frac{1}{2}\left(R_{a e b d} h^{e d}+R_{b}^{e} h_{a e}\right), \\
\mathcal{R}^{L} & =\nabla^{a} \nabla^{b} h_{a b}-\nabla^{2} h-d h .
\end{aligned}
$$

If we consider the transverse, traceless gauge $\nabla^{a} h_{a b}=0, h_{a}^{a}=0$, we have,

$$
\mathcal{R}^{L}=0, \text { and } \mathcal{R}_{a b}^{L}=-\left[\frac{1}{2} \square+d\right] h_{a b} .
$$


We can see that $\nabla^{2} R$ term will not contribute to the action. To see that $\left(\nabla_{a} \Delta R_{b c}\right)^{2}$ will also not contribute to the action, we will first carry out the linearization of $\Delta R_{a b}$ which is given by

$$
\Delta R_{a b c d}^{L}=\mathcal{R}_{a b c d}^{L}-\bar{R}_{a b c d}^{L}=\mathcal{R}_{a b c d}^{L}+\left(g_{a c}^{(0)} h_{b d}+g_{b d}^{(0)} h_{a c}-g_{a d}^{(0)} h_{b c}-g_{b c}^{(0)} h_{a d}\right) .
$$

Contracting with $g^{(0) b d}$ we get,

$$
\Delta R_{a c}^{L}=\mathcal{R}_{a c}^{L}+(d-1) h_{a c}=-\frac{1}{2}[\square+2] h_{a c} .
$$

This term vanishes on using the lowest order equation of motion for $h_{a b}$. Thus this term does not contribute to the holographic stress tensor. For the remaining $\nabla_{a} R_{b c d e}$ terms we can use the Bianchi Identity as in [29] to put the final expression [see appendix (B)] in the form (neglecting the total derivatives),

$$
\begin{aligned}
R^{b c d e} \nabla^{2} R_{b c d e}= & -4\left(\nabla_{d} R_{c e}\right)^{2}+(\nabla R)^{2}-4 R^{c d} R_{d c}^{e}{ }^{f} R_{e f}-4 R_{d}^{c} R_{f}^{d} R_{c}^{f}+2 R^{b c d e} R_{b}^{f} R_{f c d e} \\
& +2 R^{b c d e} R_{b c}^{a}{ }^{f} R_{a f d e}+4 R^{b c d e} R_{b d}^{a}{ }_{b j}^{f} R_{a c f e} .
\end{aligned}
$$

The $O\left(R^{3}\right)$ terms in the expression are given by

$S_{R^{3}}=-4 R^{c d} R_{d c}^{e}{ }^{f} R_{e f}-4 R_{d}^{c} R_{f}^{d} R_{c}^{f}+2 R^{b c d e} R_{b}^{f} R_{f c d e}+2 R^{b c d e} R_{b c}^{a}{ }^{f} R_{a f d e}+4 R^{b c d e} R_{b d}^{a}{ }_{b c}^{f} R_{a c f e}$.

Doing a similar backgroound field expansion of the above terms we have at the second order in the expansion,

$$
O\left(\Delta R^{2}\right)=4(d+2) \Delta R^{a b} \Delta R_{a b}-4(\Delta R)^{2}-2 d \Delta R^{a b c d} \Delta R_{a b c d}
$$

and at the first order there is no contribution $O(\Delta R)=0$. Hence, the coefficients that get shifted are

$$
c_{4}^{\prime}=c_{4}-8 d_{3}, \quad c_{5}^{\prime}=c_{5}+8(d+2) d_{3}, \quad c_{6}^{\prime}=c_{6}-4 d d_{3},
$$

while $c_{1}$ remains unchanged. Putting these values in the expression for $\delta T_{\mu \nu}^{\text {grav }}$, we have,

$$
\delta T_{\mu \nu}^{\text {grav }}=d \tilde{L}^{d-3}\left[c_{1}^{\prime}+2(d-2) c_{6}^{\prime}\right] h_{\mu \nu}^{(d)}=d \tilde{L}^{d-3}\left[c_{1}+2(d-2)\left(c_{6}-4 d d_{3}\right)\right] h_{\mu \nu}^{(d)} .
$$

We can also calculate the holographic stress tensor in (1.4) directly (see appendix (C)) and show the shift in the coefficient $c_{6}$ explicitly.

\section{Holographic trace anomalies}

\subsection{For $\mathcal{L}\left(g^{a b}, R_{c d e f}\right)$}

We will now calculate the holographic trace anomalies $[54,55]$ for the Lagrangian in $(1.6)$ following a simple method advocated in appendix A of [56]. This method can be easily implemented on a computer. Our results will be in agreement with [21] wherever we have been able to compare our expressions. We outline the essential steps in the computation of the anomalies. 
1. We will first choose a reference background for $g_{(0) i j}$. Since there is no restriction, we can choose any reference background, convenient for the calculation. Note that we can also use multiple reference background for $g_{(0)}$ to determine all the anomaly coefficients.

2. The form of $g_{(1) i j}$ is fixed by conformal invariance as [57]

$$
g_{(1) i j}=-\frac{1}{d-2}\left(R_{(0) i j}-\frac{R_{(0)}}{2(d-1)} g_{(0) i j}\right)
$$

where $R_{(0)}$ S are constructed out of $g_{(0)}$ respectively.

3. We will keep $g_{(2) i j}$ arbitrary. Some comments are in order. Demanding the coefficient to $g_{(2)}$ to vanish in $d=4$ in the Lagrangian enforces the condition $c_{0}=-8 c_{1}$. This is the same condition as obtained from the lowest order equations of motion. For $d=6$ the relation between $c_{0}$ and $c_{1}$ is obtained by demanding that the coefficient of $g_{(3) i j}$ vanishes. We put in $g_{(2) i j}$ for consistency but in the end it does not play a role.

4. Plugging in the FG expansion in (1.5) into (1.6), we get

$$
S=\int d z d^{d} x z^{-d-1} \sqrt{-g_{(0)}} b(x, z)
$$

where $b(x, z)=b_{0}(x)+z^{2} b_{1}(x)+\ldots$ Next we extract the coefficient of $1 / z$ term in the above term which we call $S_{l n}$.

5. The trace anomaly in $d$ dimensions is given by

$$
\left\langle T_{\mu}^{\mu}\right\rangle=b_{d / 2}
$$

where $b_{d / 2}$ is the coefficient of $z^{d}$ in the expansion for $b(x, z)$.

6. By matching the term $S_{l n}$ with the expressions for $\left\langle T_{\mu}^{\mu}\right\rangle$ we can determine various anomaly coefficients.

\subsection{1 $\mathrm{d}=2$}

In $d=2$ the $S_{l n}$ has only one anomaly term which is the Euler anomaly given by $E_{2}=\frac{1}{4} R$. Evaluated on the manifold

$$
d s^{2}=g_{(0) i j} d x^{i} d x^{j}=u\left(\chi^{2} d t^{2}+\frac{d \chi^{2}}{\chi^{2}}\right)
$$

the Euler anomaly takes the form $E_{2}=-\frac{1}{2 u}$. The $1 / \rho$ term in the action is given by $S_{l n}=-c_{1}$. Equating this with the anomaly term $\mathcal{A}=\frac{c}{8 \pi} E_{2}$ and finally putting $u=1$, we get

$$
c=16 \pi \tilde{L} c_{1}
$$




\subsection{2 $\mathrm{d}=4$}

In $d=4$ the $S_{l n}$ will contain a linear combination of the Weyl and the Euler anomalies given by

$$
\begin{aligned}
E_{4} & =R_{(0)}^{a b c d} R_{(0) a b c d}-4 R_{(0)}^{a b} R_{(0) a b}+R_{(0)}^{2}, \\
I_{4} & =R_{(0)}^{a b c d} R_{(0) a b c d}-2 R_{(0)}^{a b} R_{(0) a b}+\frac{1}{3} R_{(0)}^{2},
\end{aligned}
$$

where $R_{(0) a b c d}$ is constructed out of $g_{(0) a b}$. The trace anomaly is given by

$$
\left\langle T_{\mu}^{\mu}\right\rangle=\frac{c}{16 \pi^{2}} I_{4}-\frac{a}{16 \pi^{2}} E_{4} .
$$

We take $g_{(0)}$ as

$$
g_{(0) i j} d x^{i} d x^{j}=u\left(-\chi^{2} d t^{2}+\frac{d \chi^{2}}{\chi^{2}}\right)+\frac{v}{\theta^{2}}\left(d \theta^{2}+d \phi^{2}\right),
$$

which is of the form $A d S_{2} \times S_{2}$. In this background the anomalies take the form

$$
E_{4}=-\frac{8}{u v}, \quad I_{4}=\frac{4(u-v)^{2}}{3 u^{2} v^{2}} .
$$

The coefficient of $1 / z$ term in the action is

$$
S_{l n}=\frac{\sin \theta}{12 u v}\left(4 c_{6}(u-v)^{2}+c_{1}\left(u^{2}+4 u v+v^{2}\right)\right) .
$$

Comparing $S_{l n}$ and $\left\langle T_{\mu}^{\mu}\right\rangle$ we get, after restoring the factors of $\tilde{L}$ in $a$ and $c$

$$
a=\pi^{2} \tilde{L}^{3} c_{1}, \quad c=\pi^{2} \tilde{L}^{3}\left(c_{1}+4 c_{6}\right) .
$$

The $4 d$ holographic stress tensor in (1.4) can thus be written as

$$
\left\langle\delta T_{\mu \nu}^{\mathrm{grav}}\right\rangle=4 \tilde{L}\left[c_{1}+4 c_{6}\right] h_{\mu \nu}^{(d)}=\frac{4}{\tilde{L}^{2} \pi^{2}} c h_{\mu \nu}^{(d)} .
$$

\subsection{3 $\mathrm{d}=6$}

In $d=6$ there are four anomaly coefficients $[58,59]$ of which three are called the B-type anomalies which are the coefficients of the three Weyl anomalies and the other one is the A-type which is the coefficient of the Euler term in $6 \mathrm{~d}$. The trace anomaly in $6 d$ is given by

$$
\left\langle T_{\mu}^{\mu}\right\rangle=S_{l n}=\left(\sum_{i=1}^{3} B_{i} I_{i}+2 A E_{6}\right),
$$

where the expressions for the anomalies are given by

$$
\begin{aligned}
I_{1} & =C_{i j k l} C^{i m n j} C_{m n}{ }^{k l}, \\
I_{2} & =C_{i j}{ }^{k l} C_{k l}{ }^{m n} C_{m n}{ }^{i j}, \\
I_{3} & =C_{i j k l}\left(\nabla^{2} \delta_{j}^{i}+4 R_{j}^{i}-\frac{6}{5} R \delta_{j}^{i}\right) C^{j k l m}, \\
E_{0} & =384 \pi^{3} E_{6}=\mathcal{K}_{1}-12 \mathcal{K}_{2}+3 \mathcal{K}_{3}+16 \mathcal{K}_{4}-24 \mathcal{K}_{5}-24 \mathcal{K}_{6}+4 \mathcal{K}_{7}+8 \mathcal{K}_{8},
\end{aligned}
$$


where the terms $\mathcal{K}_{1} \ldots \mathcal{K}_{8}$ are given by (1.7). To determine the anomaly coefficients we choose for $g_{(0)}$ two manifolds $A d S_{2} \times S_{4}$ and $A d S_{2} \times S_{2} \times S_{2}$. In $A d S_{2} \times S_{4}$ we have

$$
I_{1}=-\frac{51(u-v)^{3}}{100 u^{3} v^{3}}, I_{2}=\frac{39(u-v)^{3}}{25 u^{3} v^{3}}, I_{3}=-\frac{36(19 u+v)(u-v)^{2}}{25 u^{3} v^{3}}, E_{6}=-\frac{144}{u v^{2}},
$$

while in the $A d S_{2} \times S_{2} \times S_{2}$ background we have

$$
\begin{aligned}
& I_{1}=-\frac{3\left(51 u^{3}+21 u^{2} v+17 u v^{2}-17 v^{3}\right.}{100 u^{3} v^{3}}, I_{2}=\frac{3\left(39 u^{3}-31 u^{2} v+13 u v^{2}+13 v^{3}\right)}{25 u^{3} v^{3}}, \\
& I_{3}=-\frac{12\left(11 u^{3}-39 u^{2} v+17 u v^{2}+3 v^{3}\right)}{25 u^{3} v^{3}}, E_{6}=-\frac{48}{u v^{2}} .
\end{aligned}
$$

$S_{l n}$ in the $A d s_{2} \times S_{4}$ background takes the form,

$$
S_{l n}=-\frac{9 c_{1}}{8}-\left(c_{1}+8 c_{6}\right) \frac{9(u-v)^{2}}{40 v^{2}}+\left(11 c_{1}+94 c_{6}+104 \tilde{c}_{7}-34 \tilde{c}_{8}\right) \frac{9(u-v)^{3}}{400 v^{3}}
$$

where $\tilde{c}_{7}$ and $\tilde{c}_{8}$ are coefficients of the seventh and the eighth term in (1.7). Comparing (3.17) and (3.13), we get

$$
A=\frac{3 c_{1}}{2}, \quad B_{3}=\frac{1}{128}\left(8 c_{6}+c_{1}\right), \quad 68 B_{1}-208 B_{2}+\frac{3 c_{1}}{2}+30 c_{6}+312 \tilde{c}_{7}-102 \tilde{c}_{8}=0 .
$$

Using $A d S_{2} \times S_{2} \times S_{2}$ for $g_{(0)}$ we get one more relation as,

$$
36 B_{1}-16 B_{2}+3 c_{1}+10 c_{6}+24 \tilde{c}_{7}-54 \tilde{c}_{8}=0 .
$$

We solve these two equations to get after restoring the factors of $\tilde{L}$,

$$
\begin{aligned}
A & =\frac{\tilde{L}^{5}}{2} c_{1}, & B_{3} & =\frac{\tilde{L}^{5}}{128}\left(8 c_{6}+c_{1}\right), \\
2 B_{1} & =\tilde{L}^{5}\left(-\frac{3 c_{1}}{16}-\frac{c_{6}}{2}+3 \tilde{c}_{8}\right), & 2 B_{2} & =\tilde{L}^{5}\left(-\frac{3 c_{1}}{64}+\frac{c_{6}}{8}+3 \tilde{c}_{7}\right) .
\end{aligned}
$$

The holographic stress tensor in (1.4) can now be re-expressed as,

$$
\left\langle\delta T_{\mu \nu}^{\text {grav }}\right\rangle=6 \tilde{L}^{3}\left[c_{1}+8 c_{6}\right] h_{\mu \nu}^{(6)}=6 \tilde{L}^{3} B_{3}^{\prime} h_{\mu \nu}^{(6)},
$$

where we define $B_{3}^{\prime}=128 B_{3}$. The relation between the holographic stress tensor and the asymptotic metric thus takes the form of (1.4) where $\mathcal{C}_{T}$ is related to the B-type anomaly coefficient as

$$
\mathcal{C}_{T}=f_{d} B_{3}^{\prime}
$$

\subsection{For $\mathcal{L}\left(g^{a b}, R_{c d e f}, \nabla_{a} R_{b c d e}\right)$}

We will use the same Lagrangian (2.21) for the calculation of the holographic anomalies. Here by a scaling argument as in [21] it is easy to show that the action with two covariant derivatives acting on two Riemann tensors, will take on the form as in (2.21). In the presence of the $\nabla R$ terms in the action, the central charges of the higher derivative theories get modified accordingly. 


\subsection{1 $\mathrm{d}=4$}

The additional contribution to the $S_{l n}$ is

$$
S_{(d) l n}=-\frac{16 d_{3} \sin \theta(u-v)^{2}}{3 u v},
$$

which combined with the remaining terms give

$$
S_{l n}==\frac{\sin \theta}{12 u v}\left[\left(4 c_{6}-64 d_{3}\right)(u-v)^{2}+c_{1}\left(u^{2}+4 u v+v^{2}\right)\right] .
$$

Comparing these expressions with the usual formula for the anomaly term we get the anomaly coefficients, as

$$
a=\pi^{2} \tilde{L}^{3} c_{1}, \quad c=\pi^{2} \tilde{L}^{3}\left(4 c_{6}+c_{1}-64 d_{3}\right) .
$$

We can say that $c_{6}^{\prime}=c_{6}-16 d_{3}$ and hence the holographic stress tensor of (1.4) becomes

$$
\left\langle\delta T_{\mu \nu}^{\text {grav }}\right\rangle=4 \tilde{L}\left[c_{1}+4 c_{6}^{\prime}\right] h_{\mu \nu}^{(4)}=\frac{4}{\tilde{L}^{2} \pi^{2}} c h_{\mu \nu}^{(4)},
$$

as before for $4 d$.

\subsection{2 $\mathrm{d}=6$}

In $6 d$ the additional contribution to $S_{l n}$ due to the $(\nabla R)^{2}$ terms in $A d S_{2} \times S_{4}$ is,

$$
S_{(d) l n}=-\frac{108(7 u+3 v)(u-v)^{2}}{25 u^{2} v \theta^{4}} d_{3} .
$$

Comparing the total contribution to the coefficient of $1 / z$ term with the expression for $\left\langle T_{\mu}^{\mu}\right\rangle$ for $A d S_{2} \times S_{4}$ and $A d S_{2} \times S_{2} \times S_{2}$ we get, after restoring the factors of $\tilde{L}$,

$$
\begin{aligned}
A & =\frac{3 \tilde{L}^{5}}{2} c_{1}, & B_{3} & =\frac{\tilde{L}^{5}}{128}\left(c_{1}+8 c_{6}-192 d_{3}\right), \\
2 B_{1} & =\tilde{L}^{5}\left(-\frac{3 c_{1}}{16}-\frac{c_{6}}{2}+3 \tilde{c}_{8}+24 d_{3}\right), & 2 B_{2} & =\tilde{L}^{5}\left(-\frac{3 c_{1}}{64}+\frac{c_{6}}{8}+3 \tilde{c}_{7}-6 d_{3}\right)
\end{aligned}
$$

where $c_{6}^{\prime}=c_{6}-4 d d_{3}$. The holographic stress tensor in (1.4) can now be written as

$$
\delta T_{\mu \nu}^{\mathrm{grav}}=6 \tilde{L}^{3}\left[c_{1}+8 c_{6}^{\prime}\right] h_{\mu \nu}^{(6)}=6 \tilde{L}^{3} B_{3}^{\prime} h_{\mu \nu}^{(6)},
$$

for the $6 d$ case where as before we define $B_{3}^{\prime}=128 B_{3}$.

\section{Holographic two point function for higher derivative theories in arbi- trary dimensions}

In this section we will show that the coefficient in the expression for the holographic stress tensor is related to the coefficient in the holographic two point function in arbitrary dimensions for any higher derivative theory whose bulk Lagrangian is of the form $\mathcal{L}\left(g^{a b}, R_{b c d e}, \nabla_{a} R_{b c d e}\right)$. In even dimensions the coefficient of the holographic two point 
function is related to the coefficient of the two point function in field theory which is proportional to the B-type anomaly coefficient [27, 28] (our results in six dimensions are new). The details of the calculation from the field theory side are done in appendix (D). In odd dimensions there is no anomaly. We will show that the coefficient appearing in the expression of the holographic stress tensor is related to the coefficient of the holographic two point functions in arbitrary dimensions.

As previously, we will consider the action,

$$
S=\int d^{d+1} x \sqrt{g}\left[c_{0}+c_{1} \Delta R+\frac{c_{4}}{2} \Delta R^{2}+\frac{c_{5}}{2} \Delta R^{a b} \Delta R_{a b}+\frac{c_{6}}{2} \Delta R^{a b c d} \Delta R_{a b c d}\right],
$$

where $c_{0}=-2 d c_{1}$. The advantage of using the above action for the computation of the two point function is that the result is then valid for any arbirary higher derivative theory of gravity of the form $\mathcal{L}\left(g^{a b}, R_{b c d e}, \nabla_{a} R_{b c d e}\right)$ with $c_{6}$ replaced by $c_{6}^{\prime}$ as argued previously. To compute the two point function it is sufficient to keep upto $O(\Delta R)^{2}$ terms only since as we are expanding when we expand around the AdS background, $O(\Delta R)^{3}$ terms will start at order $O\left(h^{3}\right)$. To compute the two point functions we will follow the arguments of $[25,26]$ where it is shown that to calculate the two point functions it is sufficient to look at components like $\left\langle T_{x y} T_{x y}\right\rangle$ since the other structures are completely determined by symmetry. Following $[25,26]$ we turn on a component $r^{2} h_{x y}(r, z) / L^{2}$ of the metric perturbations. The quadratic action for the fluctuation of the above form for our case is given by

$$
\begin{aligned}
S=\int & d^{d+1} x\left[K_{1} \phi^{2}+K_{2}\left(\partial_{z} \phi\right)^{2}+K_{3} \partial_{z}^{2} \phi^{2}+K_{4} \partial_{z}^{2} \phi \partial_{r} \phi+K_{5}\left(\partial_{r} \phi\right)^{2}+K_{6}\left(\partial_{r} \partial_{z} \phi\right)^{2}\right. \\
& \left.+K_{7} \partial_{r}^{2} \phi \partial_{z}^{2} \phi+K_{8} \partial_{r} \phi \partial_{r}^{2} \phi+K_{9}\left(\partial_{r}^{2} \phi\right)^{2}+K_{10} \phi \partial_{z}^{2} \phi+K_{11} \phi \partial_{r} \phi+K_{12} \phi \partial_{r}^{2} \phi\right] .
\end{aligned}
$$

The last term can be integrated by parts to obtain

$$
K_{12} \phi \partial_{r}^{2} \phi=\partial_{r}\left(K_{12} \phi \partial_{r} \phi\right)-K_{12}\left(\partial_{r} \phi\right)^{2}-\partial_{r} K_{12} \phi \partial_{r} \phi,
$$

where we have assumed that there exists a generalized Gibbons-Hawking term which takes care of the total derivatives. We will consider the scalar field to be

$$
\phi(r, z)=\phi_{k}(r) e^{-i k z}
$$

Taking the Fourier transform of the action, after the integration by parts of the last term, we have

$$
\begin{aligned}
\mathcal{A}= & \int d^{d+1} k\left[K_{1} \phi_{k} \phi_{-k}+K_{2} k^{2} \phi_{k} \phi_{-k}+K_{3} k^{4} \phi_{k} \phi_{-k}-\frac{1}{2} K_{4} k^{2} \phi_{k} \dot{\phi}_{-k}-\frac{1}{2} K_{4} k^{2} \phi_{-k} \dot{\phi}_{k}+K_{5} \dot{\phi}_{k} \dot{\phi}_{-k}\right. \\
& +K_{6} k^{2} \dot{\phi}_{k} \dot{\phi}_{-k}-\frac{1}{2} K_{7} k^{2} \ddot{\phi}_{k} \phi_{-k}+\frac{1}{2} \partial_{r}\left(K_{7} k^{2} \phi_{k}\right) \dot{\phi}_{-k}-\frac{1}{2} \partial_{r}\left(K_{8} \dot{\phi}_{k}\right) \dot{\phi}_{-k}-\frac{1}{2} \dot{K}_{8} \dot{\phi}_{k} \dot{\phi}_{-k} \\
& \left.-\partial_{r}\left(K_{9} \ddot{\phi}_{k}\right) \dot{\phi}_{-k}-K_{10} k^{2} \phi_{k} \phi_{-k}+\frac{1}{2} K_{11} \phi_{k} \dot{\phi}_{-k}+\frac{1}{2} K_{11} \phi_{-k} \dot{\phi}_{k}-K_{12} \dot{\phi}_{k} \dot{\phi}_{-k}-\dot{K}_{12} \phi_{k} \dot{\phi}_{-k}\right],
\end{aligned}
$$


where ${ }^{\circ}$ denotes derivative with respect to $r$. The terms $K_{i}$ are given by

$$
\begin{aligned}
K_{1} & =d c_{1} r^{d-1}, \quad K_{2}=\frac{3}{2} c_{1} r^{d-3}, \quad K_{3}=\left(\frac{c_{5}}{4}+c_{6}\right) r^{d-5}, \quad K_{4}=\frac{1}{2}\left[(d+1) c_{3}+4 c_{6}\right] r^{d-2}, \\
K_{5} & =\frac{1}{4}\left[6 c_{1}+(d+1)^{2} c_{5}+4(d+7) c_{6}\right] r^{d+1}, \quad K_{6}=2 c_{6} r^{d-1}, \quad K_{7}=\frac{1}{2} c_{5} r^{d-1}, \\
K_{8} & =\frac{1}{2}\left[(d+1) c_{5}+12 c_{6}\right] r^{d+2}, \quad K_{9}=\frac{1}{4}\left(c_{5}+4 c_{6}\right) r^{d+3}, \quad K_{10}=2 c_{1} r^{d-3}, \\
K_{11} & =2(d+2) c_{1} r^{d}, \quad K_{12}=2 c_{1} r^{d+1} .
\end{aligned}
$$

After integration by parts the above action can be written as a boundary term ${ }^{7}$

$$
\begin{aligned}
\partial \mathcal{A}= & -\frac{1}{2} K_{4} k^{2} \phi_{k} \phi_{-k}+K_{5} \dot{\phi}_{k} \phi_{-k}+K_{6} k^{2} \dot{\phi}_{k} \phi_{-k}+\frac{1}{2} \partial_{r}\left(K_{7} k^{2} \phi_{k}\right) \phi_{-k}-\frac{1}{2} \dot{K}_{8} \dot{\phi}_{k} \phi_{-k} \\
& -\partial_{r}\left(K_{9} \ddot{\phi}_{k}\right) \phi_{-k}-K_{12} \dot{\phi}_{k} \phi_{-k},
\end{aligned}
$$

where again 'denotes derivative with respect to $r$. The solution to $(\square+2) h_{a b}=0$ still solves the higher derivative equations. ${ }^{8}$ The solution is given by (restoring the AdS radius $\tilde{L}$ )

$$
\phi_{k}(r)=\frac{2 \tilde{L}^{4}|k|^{d / 2}}{d r^{d / 2}} K_{d / 2}\left(\frac{\tilde{L}^{2}|k|}{r}\right),
$$

where $K_{d / 2}$ is the modified Bessel function of the second kind. The normalization constant is obtained by imposing the condition that $\phi_{k}(r=\infty)=1$ and $d$ is the field theory dimension. By plugging this solution back into the surface term $\partial \mathcal{A}$ and extracting the coefficient of $k^{d}$ term in the resulting expression, we get, for $A d S_{d+1} / C F T_{d}$ after restoring the factors of $\ell_{p}$,

$$
\left\langle T_{a b}(x) T_{c d}\left(x^{\prime}\right)\right\rangle=\frac{d}{\tilde{L}^{2}} \mathcal{C}_{T} \frac{\mathcal{I}_{a b, c d}\left(x-x^{\prime}\right)}{\left|x-x^{\prime}\right|^{2 d}},
$$

where the coefficient $\mathcal{C}_{T}$ is given by

$$
\mathcal{C}_{T}=f_{d} \tilde{L}^{d-1}\left[c_{1}+2(d-2) c_{6}\right],
$$

where $f_{d}$ is the constant factor given by

$$
f_{d}=2 \frac{d+1}{d-1} \frac{\Gamma[d+1]}{\pi^{d / 2} \Gamma[d / 2]}
$$

Thus the expression for $\left\langle T_{\mu \nu}^{\text {grav }}\right\rangle$ in (1.4) becomes,

$$
\left\langle T_{\mu \nu}^{\text {grav }}\right\rangle=\frac{d}{f_{d} \tilde{L}^{2}} \mathcal{C}_{T} h_{\mu \nu}^{(d)} .
$$

Note that while we have assumed the existence of a suitable generalized Gibbons-Hawking term we have not used counterterms involving boundary curvature tensors in our calculations. We have explicitly checked, the addition of such counterterms will not alter our findings.

\footnotetext{
${ }^{7}$ We have assumed that the volume counterterm gets rid of $\phi_{k} \phi_{-k}$ terms as in [22, 61].

${ }^{8}$ See e.g. [60], alternatively we just assume that there is a massless graviton which by definition solves this equation.
} 


\section{Three point functions}

The fact that we were able to get the one point and two point functions from the background field expansion seems to suggest that the analysis can be extended to the calculation of 3point functions using the same technique. We will carry out the analysis first by considering a higher derivative Lagrangian of the form $\mathcal{L}\left(g^{a b}, \Delta R_{a b c d}\right)$ and then extending the analysis to the case where $\mathcal{L}\left(g^{a b}, \Delta R_{a b c d}, \nabla_{a} \Delta R_{b c d e}\right)$.

Direct holographic calculation of the three point functions are involved and challenging. We will follow the alternative route to derivation of the three point functions following the analysis of [23] and used in [23, 25, 26, 62-65]. The energy flux associated with a localized perturbation of fixed energy $\epsilon_{i j} T^{i j}$, where $\epsilon_{i j}$ is the polarization tensor, in the $d(>3)$ dimensional CFT background is given by

$$
\langle\epsilon(\mathbf{n})\rangle=\frac{E}{\Omega_{d-2}}\left[1+t_{2}\left(\frac{\epsilon_{i j}^{*} \epsilon_{i k} n^{j} n^{k}}{\epsilon_{i j}^{*} e_{i j}}-\frac{1}{d-1}\right)+t_{4}\left(\frac{\left|\epsilon_{i j} n^{j} n^{k}\right|^{2}}{\epsilon_{i j}^{*} e_{i j}}-\frac{2}{d^{2}-1}\right)\right] .
$$

Here $E$ is the total energy flux, $\mathbf{n}$ is the outward normal in the direction in which the flux is measured and $\Omega_{d-2}$ is the volume of a unit $S^{d-1}$ sphere. The coefficients $t_{2}$ and $t_{4}$ are determined holographically in the following way. From the holographic side we insert graviton perturbations $h_{\mu \nu}$ dual to the energy insertion in the field theory and evaluate the on-shell cubic term in the higher derivative Lagrangian corresponding to these graviton insertions. Following $[23,25,26]$, we consider the shockwave background with perturbations in $d$ dimensions:

$$
d s_{s w}^{2}=\frac{\tilde{L}^{2}}{u^{2}}\left[\delta\left(y^{+}\right) W(\vec{y}, u)\left(d y^{+}\right)^{2}-d y^{+} d y^{-}+d \vec{y}^{2}+d u^{2}\right]+h_{i j} d x^{i} d x^{j},
$$

where $\vec{y}^{2}=\sum_{i=0}^{d-2} y_{i}^{2}$ and $d$ is the dimension of the field theory. The function $W(\vec{y}, u)$ is given by

$$
W(\vec{y}, u)=\frac{2^{d-1}}{\left(1+n_{d-1}\right)^{d-1}} \frac{u^{d}}{\left(u^{2}+(\vec{y}-\vec{Y})^{2}\right)^{d-1}},
$$

where $n_{d-1}$ is the $(d-1)$ th component of the normal vector given by

$$
n_{d-1}=\left(1-n_{i}^{2}\right)^{\frac{1}{2}}, \text { and } Y^{i}=\frac{n^{i}}{1+n_{d-1}} .
$$

$W$ satisfies the following equation in any higher derivative theory of gravity [66],

$$
\partial_{u}^{2} W-\frac{d-1}{u} \partial_{u} W+\sum_{i=1}^{d-2} \partial_{y^{i}}^{2} W=0
$$

The transverse traceless gauge brings down the number of independent components of the perturbations. In $d$ dimensions we can consider the perturbation of the form $h_{y^{1} y^{2}}=$ $\tilde{L}^{2} / u^{2} \phi(\vec{y}, u)$, while $h=0=\nabla^{\mu} h_{\mu \nu}$ relates the other components as

$$
\partial_{-} h_{y^{+} y^{1}}=\frac{1}{2} \partial_{y^{2}} h_{y^{1} y^{2}}, \partial_{-} h_{y^{+} y^{2}}=\frac{1}{2} \partial_{y^{1}} h_{y^{1} y^{2}}, \partial_{-} h_{y^{+} y^{+}}=\frac{1}{4}\left(\partial_{y^{1}} h_{y^{+} y^{1}}+\partial_{y^{2}} h_{y^{+} y^{2}}\right) .
$$


It is sufficient to turn on these components only for general $d(>3)$ dimensions. The component $h_{y^{1} y^{2}}$ satisfies the lowest order equation of motion for a scalar field in the $A d S_{d+1}$ background given by,

$$
\partial_{u}^{2} \phi-\frac{d-1}{u} \partial_{u} \phi+\sum_{i=0}^{d-2} \partial_{y^{i}}^{2} \phi-4 \partial_{+} \partial_{-} \phi=0 .
$$

\section{$5.1 \mathcal{L}\left(g^{a b}, R_{c d e f}\right)$}

Using the equation of motion for $\phi$ and $W$ we can evaluate the on-shell cubic effective action to get the most general form in $d(>3)$ dimensions as ${ }^{9}$

$$
\begin{aligned}
S_{W \phi^{2}}^{(3)}= & -\frac{1}{4} \int d^{d+1} x \sqrt{-g} \phi \partial_{-}^{2} \phi\left[2\left(c_{1}+2(d-2) c_{6}\right) W-2 u\left(2 c_{6}-12 d \tilde{c}_{7}+3(3 d-4) \tilde{c}_{8}\right) \partial_{u} W\right. \\
& -24 u^{2}\left(2 \tilde{c}_{7}-\tilde{c}_{8}\right) \sum_{i>2}^{d-1} \partial_{i-2}^{2} W+u^{2}\left(2 c_{6}-12(8-d) \tilde{c}_{7}+3(12-d) \tilde{c}_{8}\right)\left(\partial_{1}^{2} W+\partial_{2}^{2} W\right) \\
& \left.-24 u^{3}\left(2 \tilde{c}_{7}-\tilde{c}_{8}\right)\left(\sum_{i=1}^{d-1} \partial_{i}^{2} \partial_{u} W-u \sum_{i>j}^{d-1} \partial_{i}^{2} \partial_{j}^{2} W\right)\right]\left.\right|_{u=1, y_{1}=0, y_{2}=0} .
\end{aligned}
$$

Note that the integral localizes on $u=1, y_{1}=0, y_{2}=0$ [23-26]. As a result we do not have to worry about boundary terms like the generalized Gibbons-Hawking term or the boundary counterterms in this calculation. Comparing with the standard form given in $[25,26]$,

$$
S_{W \phi^{2}}^{(3)}=-\frac{\mathcal{C}_{T}}{4 f_{d} \tilde{L}^{d-1}} \int d^{d+1} x \sqrt{-g} \phi \partial_{-}^{2} \phi W\left[1+t_{2} T_{2}+t_{4} T_{4}\right]
$$

and $T_{2}$ and $T_{4}$ are given by

$$
T_{2}=\frac{n_{1}^{2}+n_{2}^{2}}{2}-\frac{1}{d-1}, \quad T_{4}=2 n_{1}^{2} n_{2}^{2}-\frac{2}{d^{2}-1},
$$

while the coefficients $t_{2}, t_{4}$ are given by, ${ }^{10}$

$t_{2}=\frac{d(d-1)}{c_{1}+2(d-2) c_{6}}\left[2 c_{6}-12(3 d+4) \tilde{c}_{7}+3(7 d+4) \tilde{c}_{8}\right], \quad t_{4}=\frac{6 d\left(d^{2}-1\right)(d+2)}{c_{1}+2(d-2) c_{6}}\left(2 \tilde{c}_{7}-\tilde{c}_{8}\right)$.

This is the expected result for cubic Lovelock theory [62-65] where $2 \tilde{c}_{7}=\tilde{c}_{8}$ and hence $t_{4}=$ 0 . We have also checked that our general expressions are in agreement with [25, 26, 67, 68].

\footnotetext{
${ }^{9}$ To reach this simple form, we need to integrate by parts and use the on-shell conditions multiple number of times.

${ }^{10}$ If we set $W=1$ then we would be left with just the two point function which would be proportional to $\mathcal{C}_{T}$ as expected.
} 


\section{$5.2 \mathcal{L}\left(g^{a b}, R_{c d e f}, \nabla_{a} R_{b c d e}\right)$}

We now extend the analysis of the previous section to higher curvature theories containing covariant derivatives of the Riemann tensor. In section (2.2) we have shown how the presence of the $\nabla^{2} \Delta R^{2}$ terms modify the coefficient $c_{6} \rightarrow c_{6}^{\prime}=c_{6}-4 d d_{3}$. In addition the cubic order coefficients are modified as

$$
\begin{array}{ll}
\tilde{c}_{7} \rightarrow \tilde{c}_{7}^{\prime}=\tilde{c}_{7}-d_{3} & \tilde{c}_{8} \rightarrow \tilde{c}_{8}^{\prime}=\tilde{c}_{8}+4 d_{3}, \quad \tilde{c}_{2} \rightarrow \tilde{c}_{2}^{\prime}=\tilde{c}_{2}-4 d_{3}, \\
\tilde{c}_{5} \rightarrow \tilde{c}_{5}^{\prime}=\tilde{c}_{5}+4 d_{3}, & \tilde{c}_{6} \rightarrow \tilde{c}_{6}^{\prime}=\tilde{c}_{6}+2 d_{3} .
\end{array}
$$

Thus $c_{i}$ and $\tilde{c}_{i}$ in (5.11) will be replaced by $c_{i}^{\prime}$ and $\tilde{c}_{i}^{\prime}$ respectively. In this section we will consider additional terms like $\nabla^{2} \Delta R^{3}$ terms in the action (1.6). For $\nabla \nabla \Delta R^{3}$ terms, since the linearized Ricci tensor and scalar curvature vanishes by using the tracelessness condition and the lowest order equation of motion satisfied by $h_{a b}$, as shown in section (2.2), the terms which contribute to the three point functions are

$$
S_{3}=e_{1} R_{c d}^{a b} R_{e f}^{c d} \nabla^{2} R_{a b}^{e f}+e_{2} R_{c d}^{a b} R_{e f}^{c d} \nabla^{2} R_{a b}^{e f} .
$$

To show that these are the only tensor structures that contribute to the three point functions, consider the first term which can be shown to be,

$$
R_{c d}^{a b} R_{e f}^{c d} \nabla^{2} R_{a b}^{e f}=\nabla_{m}\left(\Delta R_{c d}^{a b} \Delta R_{e f}^{c d} \nabla^{m} \Delta R_{a b}^{e f}\right)-2 \nabla_{m} \Delta R_{c d}^{a b} \nabla^{m} \Delta R_{a b}^{e f} \Delta R_{e f}^{c d},
$$

where the overall factor of 2 comes because of $\nabla$ acting on any term other than $\nabla \Delta R$ are equivalent. Similarly it can be shown for the second term as well.

These terms have additional contribution to the coefficients $t_{2}$ and $t_{4}$ but $\mathcal{C}_{T}$ remains unaffected. The coefficients $c_{i}$ and $\tilde{c}_{i}$ in (5.11) are replaced by their effective values as,

$$
t_{2}=\frac{d(d-1)}{c_{1}+2(d-2) c_{6}^{\prime}}\left[2 c_{6}^{\prime}-12(3 d+4) \tilde{c}_{7}^{\prime \prime}+3(7 d+4) \tilde{c}_{8}^{\prime \prime}\right], t_{4}=\frac{6 d\left(d^{2}-1\right)(d+2)}{c_{1}+2(d-2) c_{6}^{\prime}}\left(2 \tilde{c}_{7}^{\prime \prime}-\tilde{c}_{8}^{\prime \prime}\right),
$$

where $\tilde{c}_{7}^{\prime \prime}=\tilde{c}_{7}^{\prime}+2 d e_{1}$ and $\tilde{c}_{8}^{\prime \prime}=\tilde{c}_{8}^{\prime}+2 d e_{2}$.

We mention here that although we leave the analysis for the general $\nabla \ldots \nabla \Delta R \ldots \Delta R$ terms for future work, we feel that this pattern will continue to persist so that the $\nabla$ terms in the action (1.6) will modify the coefficients appearing in the two and the three point functions and the form of $C_{T}, t_{2}$, and $t_{4}$ will remain the same as in (5.11) with the coefficients being replaced by similar shifted ones as discussed above.

\section{Application: $\eta / s$ for higher derivative theories}

As an application of the background field expansion method, we calculate the ratio of the shear viscosity and entropy density [43-45] for higher derivative theories [30-40]. This can be done in arbitrary dimensions but for simplicity, we will illustrate for the $d=4$ plasma. Following $[69]^{11}$ we will use the pole method to calculate the shear viscosity where only

\footnotetext{
${ }^{11}$ See also [70].
} 
the near horizon data is important. Following [69] we write the black hole metric as

$$
d s^{2}=\frac{\tilde{L}^{2}}{4 f(z)} \frac{d z^{2}}{(1-z)^{2}}+\frac{r_{0}^{2}}{\tilde{L}^{2}(1-z)}\left[-f(z) d t^{2}+\left(d x_{1}+\phi(t) d x_{2}\right)^{2}+d x_{2}^{2}+d x_{3}^{2}\right] .
$$

To compute the shear viscosity and the entropy density we need to construct the horizon perturbatively by solving the equations of motion for the higher derivative action order by order in coordinate distance from the horizon but exactly in the couplings. The solution can be written as

$$
f(z)=2 z+f_{2} z^{2}+f_{3} z^{3}+\ldots,
$$

where $f_{2}$ and $f_{3}$ are functions of the coefficients appearing in the action. The factor of 2 fixes the temperature with a particular normalization as

$$
T=\frac{r_{0}}{\pi \tilde{L}^{2}} .
$$

To compute the shear viscosity we have to plug in a perturbation $h_{x y}$ and compute the retarded Green's function

$$
G_{R}^{x y, x y}(\omega)=-i \int \theta(t)\left\langle T^{x y}(t) T^{x y}(0)\right\rangle e^{-i \omega t},
$$

and finally

$$
\eta=\lim _{\omega \rightarrow 0} \frac{\operatorname{Im} G_{R}^{x y, x y}(\omega)}{\omega} .
$$

We plug in the perturbation corresponding to the shear mode at zero momentum corresponding to the change of basis

$$
d x_{1} \rightarrow d x_{1}+\phi(t) d x_{2}
$$

Plugging this into the action (1.6), we get

$$
S_{\phi^{2}}=\int d^{5} x\left(\mathcal{A}_{1} \phi_{\omega}^{\prime} \phi_{-\omega}^{\prime}+\mathcal{A}_{2} \phi_{\omega}^{\prime \prime} \phi_{-\omega}^{\prime \prime}\right)
$$

where $\mathcal{A}_{1}$ and $\mathcal{A}_{2}$ are function of the coefficients in the action (1.6). Following [25, 26, 69], we apply the pole method for any general action of the form

$$
S_{\phi^{2}}=\int d^{d} x d z \mathcal{L}_{\phi}^{(2)}\left(\partial_{z} \phi, \partial_{t} \phi\right)
$$

using which

$$
\eta=-8 \pi T \lim _{\omega \rightarrow 0} \frac{\operatorname{Res}_{z=0} \mathcal{L}_{\phi=z^{i \omega T}}^{(2)}}{\omega^{2}} .
$$

Putting in $\phi(t)=e^{-i \omega t}$ we thus extract the coefficient of $1 / z$ term and expanding upto quadratic orders in $\omega$, we finally get,

$$
\eta=r_{0}^{3}\left(A_{1}+B_{1} f_{2}+C_{1} f_{2}^{2}+C_{3} f_{3}\right),
$$


where the coefficients $A_{1}, B_{1}, C_{1}$ and $C_{3}$ are functions of the coefficients in (1.6). Similarly the entropy density for the higher derivative action is computed using the Wald formula and takes on the form

$$
s=4 \pi r_{0}^{3}\left(A_{2}+B_{2} f_{2}+C_{2} f_{2}^{2}\right) .
$$

Note that in the above expressions for both $\eta$ and $s$, we have set the AdS radius $\tilde{L}=1$. The deviation of the $\eta / s$ ratio from the KSS bound [43-45] for the action (1.6) corresponding to the case when $t_{4}=0$ and in the absence of $O\left((\Delta R)^{3}\right)$ terms is simply given by

$$
\left(\frac{\eta}{s}-\frac{1}{4 \pi}\right) s=-2 c_{6} r_{0}^{3} .
$$

The explicit form of $\eta$ and $s$ are given in the appendix (E) for a general $R^{2}$ theory where it is shown that for particular values of the coupling constants of the general $R^{2}$ theory, the ratio can be driven to zero. As another example we quote the results for the $W^{3}$ gravity below where the lower bound for $\eta / s$ is much lower than the KSS bound.

Example: $\boldsymbol{W}^{\mathbf{3}}$ theory. In $[25,26]$, a specific six derivative theory was considered which led to equations of motion for fluctuations which were second order in radial derivatives. The motivation was to consider putting bounds on $\eta / s$ using the positive energy constraints as well as comparing these with the causality constraints. It was found that the positive energy constraints bounded the couplings and led to $(\eta / s)_{\min } \approx 0.414 / 4 \pi$. In light of our general analysis, we will consider the following six derivative Lagrangian [23] which also leads to $t_{4} \neq 0$ and we will put bounds on the couplings. [23] had already considered this action perturbatively in the couplings:

$$
S=\int d^{5} x \sqrt{g}\left[R+\frac{12}{\tilde{L}^{2}}+\frac{\tilde{L}^{2}}{2} \lambda W^{2}+\tilde{L}^{4} \mu W^{3}\right],
$$

where $W^{2}=C_{a b c d} C^{a b c d}$ and $W^{3}=C_{c d}^{a b} C_{e f}^{c d} C_{a b}^{e f}$ with $C_{a b c d}$ being the Weyl tensor. If we expand this action around the AdS background to get (1.6), then the coefficients of (1.6) for this action are given by $c_{0}=-8 c_{1}, c_{1}=1, c_{4}=\frac{\lambda}{6}, c_{5}=-\frac{4 \lambda}{3}, c_{6}=\lambda, \tilde{c}_{1}=\frac{\mu}{2}, \tilde{c}_{2}=$ $-4 \mu, \tilde{c}_{3}=\frac{8 \mu}{3}, \tilde{c}_{4}=\frac{64 \mu}{27}, \tilde{c}_{5}=-\frac{14 \mu}{9}, \tilde{c}_{6}=\frac{7 \mu}{54}, \tilde{c}_{7}=\mu$ and $\tilde{c}_{8}=0$. Note that for $W^{3}$ gravity $f_{\infty}=1$ and $\tilde{L}=1$. The coefficients $\mathcal{C}_{T}, t_{2}$ and $t_{4}$ take the form

$$
\mathcal{C}_{T}=2(1+4 \lambda), \quad t_{2}=\frac{24(\lambda-96 \mu)}{1+4 \lambda}, \quad t_{4}=\frac{4320 \mu}{1+4 \lambda} .
$$

Using the constraints for $t_{2}$ and $t_{4}$ listed in $[25,26]$, we find that $\lambda$ and $\mu$ are bounded (see figure 1). The shear viscosity and the entropy density for this action takes the form

$$
\begin{aligned}
& \eta=\frac{r_{0}^{3}}{6}\left[3-6\left(1+2 f_{2}\right) \lambda-16\left(7-40 f_{2}+16 f_{2}^{2}+36 f_{3}\right) \mu\right], \\
& s=\frac{2 \pi r_{0}^{3}}{3}\left[3+6\left(1-2 f_{2}\right) \lambda+16\left(1-2 f_{2}\right)^{2} \mu\right],
\end{aligned}
$$

where $f_{3}$ is given in terms of $f_{2}$ by,

$$
f_{3}=\frac{270-64 \mu+18 \lambda+f_{2}(216-171 \lambda+656 \mu)-6 f_{2}^{2}(9-42 \lambda+304 \mu)+4 f_{2}^{3}(9 \lambda+368 \mu)+128 f_{2}^{4} \mu}{36\left(-9-6\left(1-2 f_{2}\right) \lambda+16\left(1-2 f_{2}\right)^{2} \mu\right)},
$$




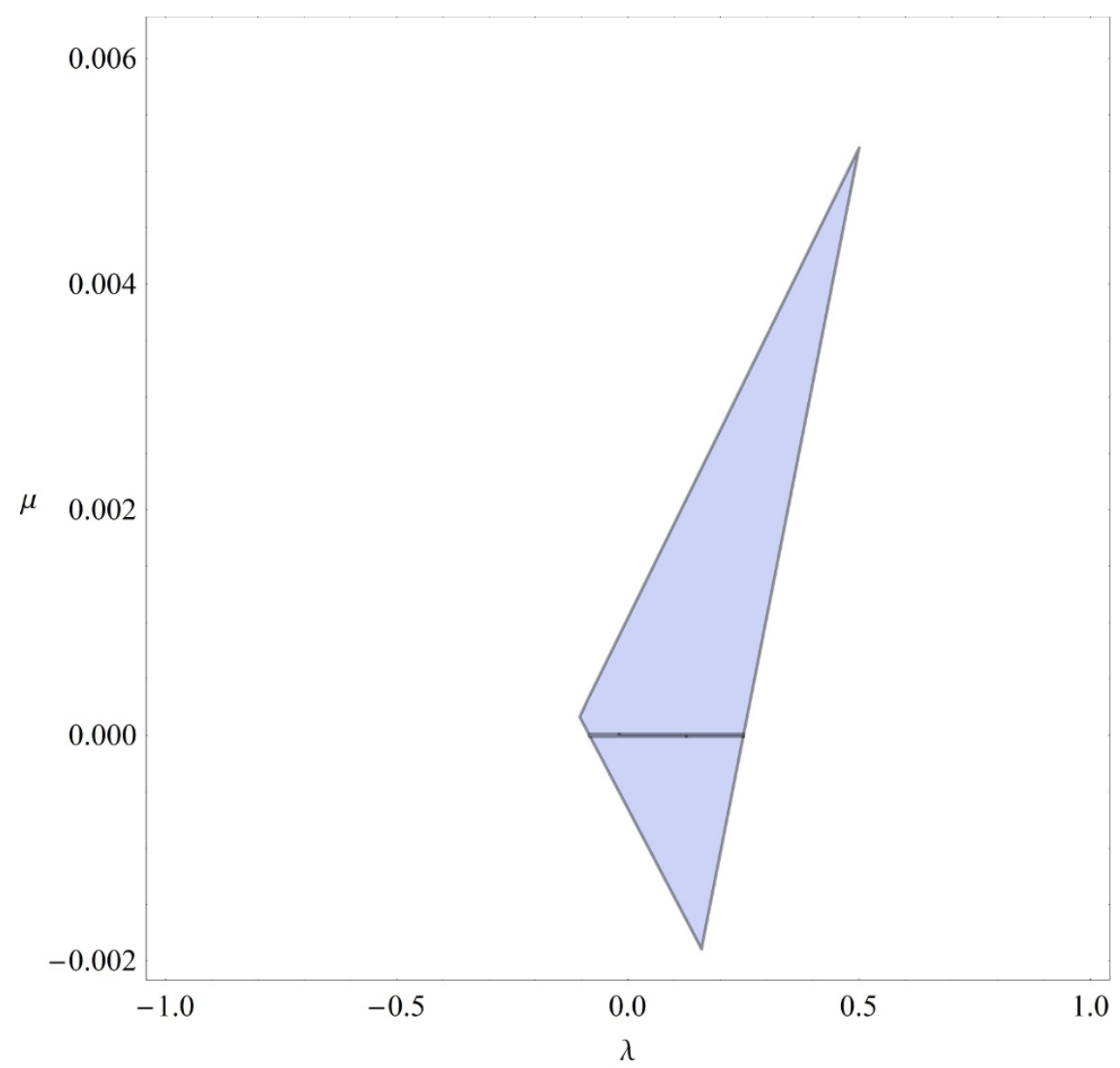

Figure 1. $\lambda$ vs $\mu$ plot. The horizontal line corresponds to $\mu=0$. $(\eta / s)_{\min }=0.55 / 4 \pi$ for $\mu=0$ and $(\eta / s)_{\min }=0.17 / 4 \pi$ for $\mu \neq 0$.

where $f_{2}$ satisfies

$$
64\left(1-2 f_{2}\right)^{3} \mu+36\left(6\left(1+f_{2}\right)-\lambda+4\left(1-f_{2}\right) f_{2} \lambda\right)=0 .
$$

This equation has three roots and we will choose the correct root as the one which for the Einstein case goes to $f_{2}=-1$. Substituting for the Einstein value of $f_{2}$ we also get that $f_{3}=0$ in the Einstein limit. We present the bounds on $\lambda$ and $\mu$ in figure(1) obtained from the causality constraints given by $[25,26]$,

$$
\begin{aligned}
\text { Tensor: } 1-\frac{1}{3} t_{2}-\frac{2}{15} t_{4} & \geq 0, \\
\text { Vector: } 1+\frac{1}{6} t_{2}-\frac{2}{15} t_{4} & \geq 0, \\
\text { Scalar: } 1+\frac{1}{3} t_{2}+\frac{8}{15} t_{4} & \geq 0 .
\end{aligned}
$$

The minimum values of $\eta / s$ for $\mu \neq 0$ lie close to the uppermost vertex of the triangle. For Weyl squared gravity $\mu=0$ and constraints give $-1 / 12<\lambda<1 / 4$. This is presented 
as a single line interval in the $\lambda-\mu$ plot. The minimum value of $\eta / s$ corresponds to $\lambda=1 / 4$ and $\mu=0$ which is at the extreme right end of the interval. The minimum value of $\eta / s$ for the $W^{3}$ gravity is given by

$$
\frac{\eta}{s} \approx \frac{0.17}{4 \pi}
$$

for $\lambda=1 / 2$, and $\mu=1 / 192$ which is the uppermost vertex of the triangle. For $\mu=0$, i.e., for Weyl squared gravity the minimum value of the ratio is $\eta / s \approx 0.55 / 4 \pi$.

Thus even though $\langle\epsilon\rangle>0$ for general $R^{2}$ theory, the $\eta / s$ ratio can be driven to zero as we show in appendix (E). Further for $W^{2}$ theory we can see that the bound goes down to about $55 \%$ of the KSS value whereas for $W^{2}+W^{3}$ theory it is $17 \%$ of the KSS bound. There are non-unitary modes in this theory. So it appears that unitarity is not a prerequisite for a bound. As in [25, 26], there could be potential plasma instabilities and it may be interesting to analyse these.

\section{Discussion}

In this paper we have computed one, two and three point functions for a general gravity Lagrangian of the form $\mathcal{L}\left(g^{a b}, R_{c d e f}, \nabla_{a} R_{b c d e}\right)$. We explained that the coefficient appearing as the proportionality between the renormalized stress tensor and the bulk metric is related to $\mathcal{C}_{T}$, the coefficient appearing in the two point function of stress tensors. Further we saw how this relates to B-type anomaly coefficients in even dimensions. We also computed three point functions for bulk Lagrangians of the above form in arbitrary dimensions.

Our general form of the action given in eq. (1.6) packages the A-type anomaly coefficient (or its analog in odd dimensions) into $c_{1}$ while $\mathcal{C}_{T}$ is given in terms of $c_{1}, c_{6}$. Again we emphasise that all these coefficients themselves depend on all higher derivative terms that appear in the original bulk Lagrangian. This simple separation of the A-type anomaly coefficient as a proportionality constant in front of $\Delta R$ makes it very tempting to think that this is a useful starting point for a general proof of holographic version of the $a$-theorem $[71,72]$ in arbitrary dimensions. We can speculate how this may work: first note that the background around which we are expanding could be either the AdS in the ultraviolet or the AdS in the infrared. This means that the respective background expanded Lagrangians must be equal to one another. If there was a matter sector as well, it makes sense to do a background expansion of this sector where we will use the background for the matter fields to be their values in AdS, for example for a scalar field this will be a constant (different constants in the UV and IR). We thus have a natural separation between the gravity sector and the matter sector - this was one of the vexing issues in the current literature on holographic c-theorems [73-81]; namely how does one define any energy condition if matter couples to the higher curvature terms. Thus we can envisage a situation where on the l.h.s. we have a term proportional to $\left(a_{U V}-a_{I R}\right) R$ plus other curvature terms while on the r.h.s. we can place the difference between the UV and IR matter Lagrangians. It is very tempting to speculate that $\left(a_{U V}-a_{I R}\right)>0$ is necessary for there to be no non-unitary modes on the l.h.s. arising from expanding $R$ which in turn is 
necessary (but may not be sufficient) so that there are no non-unitary modes in the matter Lagrangian. It will be nice to work this out in complete detail as this will shed light on how the proof of the $a$-theorem may work in arbitrary dimensions.

Another important question is to extend our methods and results to four point stress tensor correlation functions. As we pointed out in the introduction, while the A-type trace anomaly in $4 \mathrm{~d}$ is related to two point and three point functions, in higher dimensions it appears to depend on higher point correlation functions. Also in $3 \mathrm{~d}$ since there is no analog of $t_{2}$, it is unclear if the analog of the A-type trace anomaly (proportional to $c_{1}$ ) can be extracted from local correlation functions at all - this appears to be consistent with recent claims in [82]. The general forms for $t_{2}$ and $t_{4}$ that we have derived also seem to suggest that in order to relate the A-type anomaly coefficient in dimensions higher than 4 to the coefficients appearing in correlation functions will need at least four point functions. Furthermore, it could well be that the coupling constants for higher derivative theories are further constrained by considering four point functions. ${ }^{12}$ These reasons are sufficient motivation to look at the four point functions in the general gravity theories we have considered in this paper. May be the techniques developed in [83, 84] could help us out here.

It will be interesting to extend our results to completely general bulk Lagrangians of the form $\left.\mathcal{L}\left(g^{a b}, R_{c d e f}, \nabla_{a} R_{b c d e}, \nabla_{(a} \nabla_{b}\right) R_{c d e f}, \cdots\right)$. We expect that for the one, two and three point functions, the simple features we have found in this paper will continue to hold. Finally, it should be possible to extend our methods to study correlation functions which involve the massive graviton modes and $T_{\mu \nu}[85-87]$.

\section{Acknowledgments}

We thank Shamik Banerjee, Johanna Erdmenger, Daniel Grumiller, Rob Myers, Miguel Paulos and the theory groups at TIFR and HRI for useful discussions. KS thanks Daniel Grumiller and his group in Vienna for hospitality during the course of this work. AS thanks TIFR, HRI and YITP (especially Tadashi Takayanagi) for hospitality during the course of this work.

\section{A The Lagrangian in terms of [17]}

Consider the background field expanded Lagrangian given by

$$
\begin{aligned}
\mathcal{L}= & a_{0}+a_{2} \Delta R+b_{1} \Delta R^{2}+b_{2} \Delta R^{a b} \Delta R_{a b}+b_{3} \Delta R^{a b c d} \Delta R_{a b c d}+\sum_{i=1}^{8} \tilde{c}_{i} \Delta \mathcal{K}_{i} \\
& +Z^{\text {efabcdmprs }} \nabla_{e} \nabla_{f} \Delta R_{m p r s} \Delta R_{a b c d}+\ldots
\end{aligned}
$$

The Wald functional for any gravity dual following [20] is,

$$
E_{R}^{a b c d}=\frac{\partial \mathcal{L}}{\partial R_{a b c d}}-\nabla_{e}\left(\frac{\partial \mathcal{L}}{\partial \nabla_{e} R_{a b c d}}\right)+\cdots,
$$

\footnotetext{
${ }^{12}$ It will also be interesting to compare how constraints from entanglement entropy [88] compare with these ones.
} 
which for the above Lagrangian takes the form

$$
E_{R}^{a b c d}=a_{2} g^{\langle a b} g^{c d\rangle}+Y^{a b c d e f g h} \Delta R_{e f g h}-Z^{\text {efabcdmprs }} \nabla_{e} \nabla_{f} \Delta R_{m p r s}+\cdots,
$$

where $Y^{a b c d e f g h}$ is the tensor structure that comes from the second order terms in $\Delta R$ and $Z^{\text {efabcdmprs }}$ comes from the $\nabla_{a} R_{b c d e}$ terms in the Lagrangian. To connect this to eq. (6.8, $6.9,6.11)$ of [17] we need to evaluate $E_{R}^{a b c d}$ and $\delta E_{R}^{a b c d}$ around AdS space. We split the background metric as $g_{a b}=g_{(0) a b}+\Delta g_{a b}$. Then $\bar{R}_{a b c d}$ can be written as

$$
\begin{aligned}
\bar{R}_{a b c d} & =-\left(g_{a c} g_{b d}-g_{a d} g_{b c}\right)=-\left(g_{(0) a c} g_{(0) b d}-g_{(0) a d} g_{(0) b c}\right)-\left(g_{\langle(0) a b} \Delta g_{c d\rangle}\right) \\
& =R_{a b c d}^{(0)}-\left(g_{\langle(0) a b} \Delta g_{c d\rangle}\right)
\end{aligned}
$$

and

$$
\Delta R_{a b c d}=R_{a b c d}-\bar{R}_{a b c d}=\Delta^{0} R_{a b c d}+g_{\langle(0) a b} \Delta g_{c d\rangle},
$$

where $\Delta^{0} R_{a b c d}=R_{a b c d}-R_{a b c d}^{(0)}$ is the expansion around the background with only $g_{(0) a b}$. Using this relation with the fact that

$$
\begin{aligned}
Y^{\text {abcdefgh }} & =Y_{0}^{\text {abcdefgh }}+O(\Delta g), \\
Z^{\text {efadcdmprs }} & =Z_{0}^{\text {efabcdmprs }}+O(\Delta g),
\end{aligned}
$$

where $Y_{0}$ and $Z_{0}$ denote the quantities calculated with the metric $g_{(0)}$ which is the AdS metric for our purpose. To begin with, we consider an action without the $\nabla R$ terms. Then

$$
E_{R}^{a b c d}=a_{2} g^{\langle a b} g^{c d\rangle}+Y_{0}^{a b c d e f g h} \Delta^{0} R_{e f g h}+O(\Delta g),
$$

where we have used the tensor structure of $Y$ as

$$
Y^{a b c d e f g h}=b_{1} g^{a c} g^{b d} g^{e g} g^{f h}+b_{2} g^{a c} g^{e g} g^{b f} g^{d h}+b_{3} g^{a e} g^{b f} g^{c g} g^{d h}
$$

and when evaluated around the AdS background we have at the leading order

$$
E_{R}^{a b c d}=a_{2} g_{0}^{\langle a b} g_{0}^{c d\rangle}
$$

Comparing with eq. (6.8) of [17] we have $a_{2}=c_{1}$. Next we compute

$$
\frac{\partial E_{R}^{a b c d}}{\partial g^{e f}}=2 c_{1} h^{\langle a b} g^{c d\rangle}+\frac{\partial}{\partial g^{e f}}(Y \Delta R) .
$$

The last term gives around the AdS background

$$
\frac{\partial}{\partial g^{e f}}(Y \Delta R)=\frac{\partial Y}{\partial g} \Delta^{0} R+\frac{\partial}{\partial g^{m n}}\left(Y_{0}^{a b c d e f g h} g_{\langle(0) e f} \Delta g_{g h\rangle}\right) .
$$

The first term vanishes when evaluated on AdS and thus

$$
\left.\frac{\partial}{\partial g^{e f}}(Y \Delta R)\right|_{\text {AdS }} h^{e f}=2\left(2 d b_{1}+b_{2}\right) h g^{\langle a b} g^{c d\rangle}+2\left((d-1) b_{2}+4 b_{3}\right) h^{\langle a b} g^{c d\rangle} .
$$


Further

$$
\left.\frac{\partial E_{R}^{a b c d}}{\partial R^{e f g h}}\right|_{\text {AdS }} \delta R_{e f g h}=Y_{0}^{a b c d e f g h} \delta R_{e f g h}=b_{1} \mathcal{R} g^{\langle a b} g^{c d\rangle}+b_{2} \mathcal{R}^{\langle a b} g^{c d\rangle}+b_{3} \mathcal{R}^{a b c d},
$$

Comparing with eq. (6.11) of [17] we get $b_{1}=c_{4} / 2, b_{2}=c_{5} / 2, b_{3}=c_{6} / 2$. Further,

$$
c_{2}=-2 d c_{4}-c_{5}, \quad c_{3}=2 c_{1}-(d-1) c_{5}-4 c_{6} .
$$

The Lagrangian (1.6) thus can be written as,

$$
\begin{aligned}
S=\int d^{d+1} x \sqrt{g}[ & c_{0}+c_{1} \Delta R+\frac{c_{4}}{2} \Delta R^{2}+\frac{c_{5}}{2} \Delta R^{a b} \Delta R_{a b}+\frac{c_{6}}{2} \Delta R^{a b c d} \Delta R_{a b c d}+\sum_{i=1}^{8} \tilde{c}_{i} \Delta \mathcal{K}_{i} \\
& +Z \Delta R \nabla \nabla \Delta R+\cdots]
\end{aligned}
$$

\section{B Details of calculation for section (2.2)}

The Bianchi identity reads

$$
\nabla_{a} R_{b c d e}+\nabla_{b} R_{c a d e}+\nabla_{c} R_{a b d e}=0
$$

Then

$$
\nabla^{2} R_{b c d e}=\nabla^{a} \nabla_{b} R_{a c d e}-\nabla^{a} \nabla_{c} R_{a b d e}
$$

Using

$$
\nabla^{a} \nabla_{b} R_{a c d e}=\nabla_{b} \nabla^{a} R_{a c d e}+R_{b}^{f} R_{a c d e}+R_{b c}^{a}{ }^{f} R_{a f d e}+R_{b d}^{a}{ }^{f} R_{a c f e}+R_{b e}^{a}{ }^{f} R_{a c d f},
$$

we have

$R^{b c d e} \nabla^{2} R_{b c d e}=2 R^{b c d e} \nabla_{b} \nabla^{a} R_{a c d e}+2 R^{b c d e} R_{b}^{f} R_{f c d e}+2 R^{b c d e} R_{b c}^{a}{ }^{f} R_{a f d e}+4 R^{b c d e} R_{b d}^{a}{ }_{b c f e}$.

Again using the Bianchi Identity,

$$
\nabla^{a} R_{a c d e}=\nabla_{d} R_{c e}-\nabla_{e} R_{c d}
$$

we can write, neglecting the total derivatives

$$
R^{b c d e} \nabla^{2} R_{b c d e}=4 R^{b c d e} \nabla_{b} \nabla_{d} R_{c e}+2 R^{b c d e} R_{b}^{f} R_{f c d e}+2 R^{b c d e} R_{b c}^{a}{ }^{f} R_{a f d e}+4 R^{b c d e} R_{b d}^{a}{ }^{f} R_{a c f e}
$$

The first term can be written as (neglecting total derivatives),

$$
4 R^{b c d e} \nabla_{b} \nabla_{d} R_{c e}=-4 \nabla_{b} R^{b c d e} \nabla_{d} R_{c e}=-4\left(\nabla_{d} R_{c e}\right)^{2}-4 R^{c d} \nabla^{e} \nabla_{d} R_{c e}
$$

and

$$
-4 R^{c d} \nabla^{e} \nabla_{d} R_{c e}=(\nabla R)^{2}-4 R^{c d} R_{d c}^{e}{ }^{f} R_{e f}-4 R_{d}^{c} R_{f}^{d} R_{c}^{f}
$$




\section{Holographic stress tensor involving $\nabla R$ terms}

Here we consider an extended analysis of [17] to derive the holographic stress tensor including the $\nabla \ldots \nabla R$ terms in the action. The most general analysis is deferred for future work although from the following analysis it will be clear that the most general case will also work out in an analogous way. We consider the most general term involving two $\nabla \mathrm{s}$ in the action. Such terms after background field expansion are schematically given by

$$
S=\int d^{d+1} x \sqrt{g} Z \nabla \nabla(\Delta R)^{n},
$$

where $Z$ contains all the relevant tensor structures. Note that the Wald functional obtained from such a terms will be of the form

$$
E_{R}^{a b c d}=\cdots+Z^{e f \cdots} \nabla_{e} \nabla_{f}(\Delta R)^{n-1}+\ldots
$$

For $n>2$, these terms vanish since when we put the background AdS, $\Delta R_{a b c d}$ vanishes in the variation of $E_{R}^{a b c d}$. So the only terms at the two $\nabla \mathrm{s}$ order relevant for the calculation of the holographic stress tensor are schematically given by $\nabla \Delta R \nabla \Delta R$. These terms in the action are:

$$
S_{\nabla R}=\int d^{d+1} x \sqrt{g} Z^{\text {fabcdmnrs }} \Delta R_{m n r s} \nabla_{e} \nabla_{f} \Delta R_{a b c d},
$$

where as before $Z^{\text {efabcdmnrs }}$ contains all possible tensor structures.

We now focus on the derivation of the holographic stress tensor for the action including (2.21). The Wald functional corresponding to this term is given by

$$
E_{\nabla R}^{a b c d}=d_{1} g^{\langle a b} g^{c d\rangle} \nabla^{2} \Delta R+d_{2} \nabla^{2} \Delta R^{\langle a b} g^{c d\rangle}+d_{3} \nabla^{2} \Delta R^{a b c d},
$$

and evaluated on the AdS, $E_{\nabla R}^{a b c d}=0$, while the linearized variation of the wald function is given by

$$
\delta E_{\nabla R}^{a b c d}=\delta\left(\mathcal{Z}^{\text {efabcdmnrs }} \nabla_{e} \nabla_{f} \Delta R_{m n r s}\right),
$$

where the structure of $\mathcal{Z}$ for the contributing terms is given by

$$
\mathcal{Z}^{\text {efabcdmnrs }}=g^{e f}\left(d_{1} g^{a c} g^{b d} g^{m r} g^{n s}+d_{2} g^{a c} g^{m r} g^{b n} g^{d s}+d_{3} g^{a m} g^{b n} g^{c r} g^{d s}\right) .
$$

All indices are raised or lowered with respect to the background AdS metric $g_{a b}$. Thus combined with the original expressions in [17] for $E_{R}^{(1) a b c d}=E_{R}^{a b c d}+E_{\nabla R}^{a b c d}=E_{R}^{a b c d}$ and $\delta E_{R}^{(1) a b c d}$ is given by,

$$
\delta E_{R}^{(1) a b c d}=-c_{2} h g^{\langle a b} g^{c d\rangle}-c_{3} h^{\langle a b} g^{c d\rangle}+c_{4} \mathcal{R} g^{\langle a b} g^{c d\rangle}+c_{5} \mathcal{R}^{\langle a b} g^{c d\rangle}+c_{6} \mathcal{R}^{a b c d}+\delta E_{\nabla R}^{a b c d} .
$$

\section{C.1 $\mathrm{d}=4$}

The coefficients (2.13) for $d=4$ are given by

$$
\begin{array}{ll}
A=-\frac{c_{3}}{4}-\frac{3 c_{5}}{4}-5 c_{6}+32 d_{3}, & B=-\frac{c_{2}}{2}-2 c_{4}-c_{5}-12 d_{2}+4 d_{3}, \\
C=-c_{2}-4 c_{4}+c_{5}+32 d_{3}, & D=-c_{3}-3 c_{5}+4 c_{6}-64 d_{3} .
\end{array}
$$


Thus the coefficients $A_{1} A_{2}$ take the form

$$
\begin{aligned}
& A_{1}=-24\left(c_{6}-8 d_{3}\right) R^{2}+\frac{1}{2}\left(c_{1}-c_{3}-3 c_{5}-68 c_{6}+512 d_{3}\right)\left(\frac{r^{2}}{3}-R^{2}\right), \\
& A_{2}=\left(\frac{c_{1}}{2}-c_{2}-4 c_{4}-2 c_{5}-24 d_{2}+8 d_{3}\right) R^{2}+\frac{r^{2}}{6}\left(c_{1}-c_{3}+15 c_{5}+4 c_{6}+144 d_{2}+80 d_{3}\right) .
\end{aligned}
$$

We can use the tracelessness condition of $h_{\mu \nu}$ viz. $h_{\mu}^{(d) \mu}=0$ to eliminate $A_{2}$ and thus integrate over $A_{1}$ to get

$$
\delta S_{B}^{\mathrm{Wald}}=\frac{8 \pi \Omega_{2} \tilde{L} R^{4}}{15}\left(c_{1}+8 c_{6}-64 d_{3}\right)
$$

where $\Omega_{2}$ is the volume of the unit $S_{2}$ and finally using (2.5), we have

$$
\delta T_{t t}^{\text {grav }}=4 \tilde{L}\left[c_{1}+4\left(c_{6}-16 d_{3}\right)\right]
$$

\section{C.2 $\mathrm{d}=6$}

The corresponding coefficients in (2.13) for $d=6$ after putting $\Delta=d$ are given as

$$
\begin{aligned}
A & =\frac{1}{4}\left(-c_{3}-5 c_{5}-52 c_{6}+576 d_{3}\right), & B & =-\frac{c_{2}}{2}-3 c_{4}-\frac{11}{4} c_{5}-30 d_{2}+6 d_{3}, \\
C & =-c_{2}-6 c_{4}+2 c_{5}-60 d_{2}, & D & =-c_{3}-5 c_{5}+8 c_{6}-144 d_{3} .
\end{aligned}
$$

Putting these in the integral we have

$$
\begin{aligned}
& A_{1}=\frac{1}{2}\left(c_{1}-c_{3}-5 c_{5}-292 c_{6}+3456 d_{3}\right)\left(\frac{r^{2}}{5}-R^{2}\right)-120\left(c_{6}-12 d_{3}\right) R^{2}, \\
& A_{2}=\frac{1}{2}\left(c_{1}-2 c_{2}-12 c_{4}-11 c_{5}-120 d_{2}+24 d_{3}\right) R^{2}+\frac{1}{2}\left(c_{1}-c_{3}+70 c_{5}+8 c_{6}-264 d_{3}\right) \frac{r^{2}}{5} .
\end{aligned}
$$

Again by using the tracelessness argument we can set $h=0$ and integrating and finally using (2.5), we get,

$$
\delta T_{t t}^{\text {grav }}=\frac{35}{2 \pi \Omega_{4}} \lim _{R \rightarrow 0}\left(\frac{1}{R^{6}} \delta S_{B}^{\text {Wald }}\right)=6 \tilde{L}^{3}\left[c_{1}+8\left(c_{6}-24 d_{3}\right)\right] .
$$

\section{$\mathrm{D}\left\langle\boldsymbol{T}_{\mu \nu}(x) \boldsymbol{T}_{\rho \sigma}(0)\right\rangle$ in even dimensions}

The B-type anomaly coefficients appearing in the expression for the holographic stress tensor in even dimensions are precisely the coefficients of the stress tensor two point functions from the field theory perspective. The $2 \mathrm{~d}$ and $4 \mathrm{~d}$ cases were worked out in [28]. We will extend this result to $6 \mathrm{~d}$ in what follows. Before that we will review the $2 \mathrm{~d}$ and $4 \mathrm{~d}$ results.

The starting point of the derivation is the renormalization group equation in $[27,28]$ which takes on the form in general $d$ dimensions as

$$
\left(\mu \partial_{\mu}+2 \int d^{d} x g^{\mu \nu} \frac{\delta}{\delta g^{\mu \nu}}\right) W=0
$$


We know that

$$
\int d^{d} x g^{\mu \nu} \frac{\delta}{\delta g^{\mu \nu}} W=\int d^{d} x g^{\mu \nu}\left\langle T_{\mu \nu}\right\rangle=\int d^{d} x A_{\text {anomaly }}
$$

which gives us

$$
\mu \partial_{\mu} W=-2 \int d^{d} x A_{\text {anomaly }} .
$$

We now functionally differentiate the l.h.s. w.r.t. $g^{\mu \nu}$ twice to get

$$
\mu \partial_{\mu}\left\langle T_{a b}(x) T_{c d}(0)\right\rangle=-2 \int d^{d} x \frac{\delta^{2} A_{\text {anomaly }}}{\delta g^{a b} \delta g^{c d}} .
$$

From the general conformal properties of the 2 point functions the r.h.s. now takes the form

$$
\mu \partial_{\mu}\left\langle T_{a b}(x) T_{c d}(0)\right\rangle=\frac{C_{T}}{4(d-2)^{2} d(d+1)} \Delta_{a b c d}^{T} \mu \partial_{\mu} \frac{1}{x^{2 d-4}},
$$

where the tensor $\Delta_{a b c d}^{T}$ now takes the form

$$
\Delta_{a b c d}^{T}=\frac{1}{2}\left(S_{a c} S_{b d}+S_{a d} S_{b c}\right)-\frac{1}{d-1} S_{a b} S_{c d}, \quad \Delta_{a a c d}^{T}=0,
$$

where $S_{a b}=\partial_{a} \partial_{b}-\delta_{a b} \partial^{2}$. In general $x^{-2 d+4}$ is singular function. We need to regularize the function in what follows.

\section{D.1 $\mathrm{d}=2$}

We consider the anomaly in $d=2$ which is given by $E_{2}=\frac{1}{4} R, I_{2}=0$. The RG equation is given by

$$
\mu \partial_{\mu} W+\int d^{2} x\left\langle T_{i}^{i}\right\rangle=0
$$

and the 2 pt function is given by

$$
\mu \partial_{\mu}\left\langle T_{a b}(x) T_{c d}(0)\right\rangle=-\frac{1}{4} \int d^{2} x \frac{\delta^{2} R}{\delta g^{a b} \delta g^{c d}} .
$$

From the second order variation of $R, \delta^{2} R=h \partial^{2} h-h \partial_{e} \partial_{f} h^{e f}$ we get,

$$
\frac{\delta^{2} R}{\delta g^{a b} \delta g^{c d}}=\left[\left(g_{a b} \partial_{c} \partial_{d}+g_{c d} \partial_{a} \partial_{b}\right)-g_{a b} g_{c d} \partial^{2}\right] \delta^{2}(x) .
$$

Converting into the momentum space we can see that

$$
\mu \partial_{\mu}\left\langle T_{a b}(p) T_{c d}(0)\right\rangle=-\frac{1}{4}\left[\left(g_{a b} p_{c} p_{d}+g_{c d} p_{a} p_{b}\right)-g_{a b} g_{c d} p^{2}\right]
$$

using which we see that $\mathcal{C}_{T}$ and $c$ are proportional to one another. 


\section{D.2 $\mathrm{d}=4$}

In $4 \mathrm{~d}$ there are two anomalies given by

$$
\begin{aligned}
E_{4} & =R^{a b c d} R_{a b c d}-4 R^{a b} R_{a b}+R^{2}, \\
I_{4} & =E_{4}+2\left(R^{a b} R_{a b}-\frac{1}{3} R^{2}\right) .
\end{aligned}
$$

The contribution from the $E_{4}$ term in $4 d$ is given by the integral of

$$
\int d^{d} x \mathcal{A}_{\rho \sigma, \alpha \beta}^{E}(x-y, x-z),
$$

where the term $\mathcal{A}_{\rho \sigma, \alpha \beta}^{E}(x-y, x-z)$ is given by

$$
\begin{aligned}
& \mathcal{A}_{\rho \sigma, \alpha \beta}^{E}(x-y, x-z)= \\
& \quad-\left(\epsilon_{\sigma \alpha \gamma \kappa} \epsilon_{\rho \beta \delta \lambda} \partial_{\kappa} \partial_{\lambda}\left(\partial_{\gamma} \delta^{d}(x-y) \partial_{\delta} \delta^{d}(x-z)\right)+\epsilon_{\rho \alpha \gamma \kappa} \epsilon_{\sigma \beta \delta \lambda} \partial_{\kappa} \partial_{\lambda}\left(\partial_{\gamma} \delta^{d}(x-y) \partial_{\delta} \delta^{d}(x-z)\right)\right) .
\end{aligned}
$$

To compute the integral we first convert the $\delta^{d}(x-y)$ into momentum space and carry out the differentiations as

$$
\begin{aligned}
\partial_{\delta} \delta^{d}(x-z) \partial_{\gamma} \delta^{d}(x-y) & =\partial_{\gamma}\left(\int e^{i p(x-y)} d^{d} p\right) \partial_{\delta}\left(\int e^{i q(x-z)} d^{d} q\right) \\
& =-p_{\gamma} q_{\delta} \int e^{i(p+q) x-i p y-i q z} d^{d} p d^{d} q .
\end{aligned}
$$

Acting $\partial_{\kappa} \partial_{\lambda}$ on this, we get

$$
\partial_{\kappa} \partial_{\lambda}\left(-p_{\gamma} q_{\delta} \int e^{i(p+q) x-i p y-i q z} d^{d} p d^{d} q\right)=p_{\gamma} q_{\delta}(p+q)_{\lambda}(p+q)_{\kappa} \int e^{i(p+q) x-i p y-i q z} d^{d} p d^{d} q .
$$

Thus the first term on the l.h.s. in the above integral (D.13) becomes

$$
\epsilon_{\sigma \alpha \gamma \kappa} \epsilon_{\rho \beta \delta \lambda} p_{\gamma} q_{\delta}(p+q)_{\lambda}(p+q)_{\kappa} \int e^{i(p+q) x} d^{d} x \int e^{-i p y-i q z} d^{d} p d^{d} q,
$$

which becomes after substituting the delta function from the first integral as

$$
\epsilon_{\sigma \alpha \gamma \kappa} \epsilon_{\rho \beta \delta \lambda} \int p_{\gamma} q_{\delta}(p+q)_{\lambda}(p+q)_{\kappa} \delta^{d}(p+q) e^{-i p y-i q z} d^{d} p d^{d} q .
$$

Thus this integral vanishes on its own. Similarly it can be shown that the second part of the integral also vanishes by itself. Thus there is no contribution from the $E_{4}$ term to the anomaly. The only contribution to the anomaly comes from the term $R^{a b} R_{a b}-\frac{1}{3} R^{2}$ term in the Weyl anomaly. Thus

$$
\mu \partial_{\mu}\left\langle T_{a b}(x) T_{c d}(0)\right\rangle=-\int d^{4} x \frac{\delta^{2}}{\delta g^{a b} g^{c d}}\left[2\left(R^{m n} R_{m n}-\frac{1}{3} R^{2}\right)\right] .
$$

The last term on the r.h.s. gives

$$
\frac{\delta^{2} R^{2}}{\delta g^{a b} \gamma^{c d}}=2 \frac{\delta R}{\delta g^{a b}} \frac{\delta R}{\delta g^{c d}}
$$


After linearization of the scalar and functionally differentiating w.r.t. $g^{a b}$ we have

$$
\frac{\delta R}{\delta g^{a b}}=\left(\partial_{a} \partial_{b}-g_{a b} \partial^{2}\right) \delta^{4}(x)=S_{a b} \delta^{4}(x),
$$

where we define $S_{a b}=\partial_{a} \partial_{b}-g_{a b} \partial^{2}$. Thus the last term becomes after some integration by parts

$$
\frac{\delta^{2} R^{2}}{\delta g^{a b} \delta g^{c d}}=S_{a b} S_{c d} \delta^{4}(x)
$$

The first term on the r.h.s. becomes after integration by parts as

$$
\frac{\delta R^{m n}}{\delta g^{a b}} \frac{\delta R_{m n}}{\delta g^{c d}}=\frac{1}{2}\left(S_{a c} S_{b d}+S_{a d} S_{b c}\right) \delta^{4}(x) .
$$

Thus the total contribution from the Weyl anomaly is given by

$$
\mu \partial_{\mu}\left\langle T_{a b}(x) T_{c d}(0)\right\rangle=-4 \beta \Delta_{a b c d}^{T} \delta^{4}(x),
$$

where we define $\Delta_{a b c d}^{T}=\frac{1}{2}\left(S_{a c} S_{b d}+S_{a d} S_{b c}\right)-\frac{1}{3} S_{a b} S_{c d}$.

Thus in $4 d$ we have using $\beta=-c / 16 \pi^{2}$ from [27]

$$
\mu \partial_{\mu}\left\langle T_{a b}(x) T_{c d}(0)\right\rangle=\frac{c}{4 \pi^{2}} \Delta_{a b c d}^{T} \delta^{4}(x) .
$$

Comparing with (D.5) we have

$$
\frac{C_{T}}{4(d-2)^{2} d(d+1)} \mu \partial_{\mu} \frac{1}{x^{4}}=\frac{c}{4 \pi^{2}} \delta^{4}(x) .
$$

In $4 \mathrm{~d}$ the regularized $1 / x^{4}$ can be expressed as

$$
\mathcal{R} \frac{1}{x^{4}}=-\frac{1}{4} \partial^{2} \frac{1}{x^{2}}\left(\log \mu^{2} x^{2}\right) \Rightarrow \mu \partial_{\mu} \mathcal{R} \frac{1}{x^{4}}=2 \pi^{2} \delta^{4}(x) .
$$

Putting this in (D.25) we have

$$
c=\frac{\pi^{4}}{40} C_{T}
$$

\section{D.3 $d=6$}

In $6 \mathrm{~d}$ it is rather easy to see why only the $B_{3}$ coefficient gets picked up by the $2 \mathrm{pt}$ functions. If we look at the structures of the anomalies then only $I_{3}$ has a structure of the form

$$
I_{3} \sim C^{a b c d} \partial^{2} C_{a b c d}
$$

This makes $I_{3}$ to start at the order $O\left(h^{2}\right)$ and contributes in the $2 \mathrm{pt}$ function. While all the other anomalies start at $O\left(h^{3}\right)$ and thus do not contribute.

In $6 \mathrm{~d}$ the only contribution to the two point function comes from the term $I_{3} \sim$ $C^{a b c d} \partial^{2} C_{a b c d}$, since the other anomalies start at $O\left(h^{3}\right)$. Thus from (D.5) we have

$$
\mu \partial_{\mu}\left\langle T_{a b}(x) T_{c d}(0)\right\rangle=4 B_{3} \Delta_{a b c d}^{T} \partial^{2} \delta^{6}(x)=\Delta_{a b c d}^{T} \frac{C_{T}}{2^{7} \times 3 \times 7} \mu \partial_{\mu} \frac{1}{x^{8}} .
$$


In $6 \mathrm{~d}$ we regularize as

$$
\mathcal{R} \frac{1}{x^{8}}=-\frac{1}{96} \partial^{4} \frac{1}{x^{4}}\left(\log \mu^{2} x^{2}\right) \Rightarrow \mu \partial_{\mu} \mathcal{R} \frac{1}{x^{8}}=-\frac{1}{48} \partial^{4} \frac{1}{x^{4}} .
$$

The term on the r.h.s. for $6 \mathrm{~d}$ can be reduced to $\partial^{4} \frac{1}{x^{4}}=-4 \pi^{3} \partial^{2} \delta^{6}(x)$ and hence the r.h.s. becomes

$$
\mu \partial_{\mu} \mathcal{R} \frac{1}{x^{8}}=-\frac{1}{48} \partial^{4} \frac{1}{x^{4}}=\frac{\pi^{3}}{12} \partial^{2} \delta^{6}(x) .
$$

Thus in $6 \mathrm{~d}$ we have

$$
\mu \partial_{\mu}\left\langle T_{a b}(x) T_{c d}(0)\right\rangle=4 B_{3} \Delta_{a b c d}^{T} \partial^{2} \delta^{6}(x)=\frac{\pi^{3}}{7 \times 3^{2} \times 2^{11}} \Delta_{a b c d}^{T} C_{T} \partial^{2} \delta^{6}(x) .
$$

Hence we have

$$
B_{3}=\frac{\pi^{3}}{7 \times 3^{2} \times 2^{11}} C_{T}
$$

\section{E $\quad \eta / s$ for general $R^{2}$ theories}

We will calculate the ratio of the shear viscosity to the entropy density for four derivative theory of gravity in $d=4$ where $d$ is the boundary dimension. We want to express the ratio in terms of the field theory variables as $t_{2}$ etc. This analysis can be extended for general higher derivative theories of gravity in arbitrary dimensions. To proceed we will follow the analysis of [69] where the horizon is first constructed perturbatively and then the pole method was used to extract the shear viscosity. We first consider the metric as

$$
d s^{2}=\frac{\tilde{L}^{2}}{4 f(z)} \frac{d z^{2}}{(1-z)^{2}}+\frac{r_{0}^{2}}{\tilde{L}^{2}(1-z)}\left[-\frac{f(z)}{f_{\infty}} d t^{2}+\left(d x_{1}+\phi(t) d x_{2}\right)^{2}+d x_{2}^{2}+d x_{3}^{2}\right] .
$$

where $\phi(t)=e^{-i \omega t}$ is the fluctuation and

$$
f(z)=2 z+f_{2} z^{2}+f_{3} z^{3}+\ldots
$$

We consider the general $R^{2}$ action given by

$$
S=\int d^{5} x \sqrt{g}\left[R+\frac{12}{L^{2}}+\frac{L^{4}}{2}\left(\lambda_{1} R^{a b c d} R_{a b c d}+\lambda_{2} R^{a b} R_{a b}+\lambda_{3} R^{2}\right)\right] .
$$

To obtain the coefficients $f_{2}, f_{3}$ we plug in (E.2) into the equations of motion for the action (E.3) and solve perturbatively near the horizon. The solution for $f_{\infty}$ taking $L=$ $\tilde{L} \sqrt{f_{\infty}}$ given by,

$$
1-f_{\infty}+\frac{1}{3}\left(\lambda_{1}+2 \lambda_{2}+10 \lambda_{3}\right) f_{\infty}^{2}=0 .
$$

The expression for $c_{1}$ is given by

$$
c_{1}=\frac{1}{16 \pi^{2}}\left[1-2 f_{\infty}\left(\lambda_{1}+2 \lambda_{2}+10 \lambda_{3}\right)\right]
$$


We also express $c_{6}=\frac{\lambda_{1}}{16 \pi} f_{\infty}$. The shear viscosity and entropy density in terms of these couplings are given by

$$
\begin{aligned}
\eta & =\mathcal{C}_{1}\left[8 \lambda_{1}^{2}+\lambda_{2}+4 \lambda_{3}-20 \lambda_{2} \lambda_{3}-64 \lambda_{3}^{2}+12 \lambda_{1}\left(\lambda_{2}+2 \lambda_{3}\right)+\sqrt{\mathcal{F}}\right], \\
s & =\mathcal{C}_{2}\left[16 \lambda_{1}^{2}+\lambda_{2}+4 \lambda_{3}+20 \lambda_{2} \lambda_{3}+48 \lambda_{1} \lambda_{3}-20 \lambda_{2} \lambda_{3}-64 \lambda_{3}^{2}+\sqrt{\mathcal{F}}\right],
\end{aligned}
$$

where the normalizations are $\mathcal{C}_{2}=\frac{2 \pi r_{0}^{3} f_{\infty}^{3 / 2}}{\ell_{p}^{3}}$ and $\mathcal{C}_{1}=\frac{r_{0}^{3} f_{\infty}^{3 / 2}}{2 \ell_{p}^{3}}$ and we have set $\tilde{L}=1$.

$$
\begin{aligned}
\mathcal{F}= & \left(2 \lambda_{1}+\lambda_{2}+2 \lambda_{3}\right)\left[\left(2 \lambda_{1}+\lambda_{2}+2 \lambda_{3}\right)\left(1-12 \lambda_{1}-16 \lambda_{2}-52 \lambda_{3}\right)^{2}\right. \\
& -16\left(\lambda_{1}+\lambda_{2}+2 \lambda_{3}\right)\left(22 \lambda_{1}^{2}-\lambda_{2}\left(1-12 \lambda_{2}\right)-2 \lambda_{3}+62 \lambda_{2} \lambda_{3}+70 \lambda_{3}^{2}\right. \\
& \left.\left.-2 \lambda_{1}\left(1-19 \lambda_{2}-58 \lambda_{3}\right)\right)\right] .
\end{aligned}
$$

The corresponding expression for $t_{2}$ is $d=4$ is given by

$$
t_{2}=\frac{24 c_{6}}{c_{1}+4 c_{6}}=\frac{24 \lambda_{1} f_{\infty}}{1+2\left(\lambda_{1}-2 \lambda_{2}-10 \lambda_{3}\right) f_{\infty}} .
$$

Note that in the limit $\lambda_{1}, \lambda_{2}, \lambda_{3} \rightarrow 0$ we retrieve the result

$$
\frac{\eta}{s}=\frac{1}{4 \pi} .
$$

Note also that it is possible to make $\eta / s$ arbitrarily small by tuning the values of $\lambda$ s'. For example for $\lambda_{1}=0.31517, \lambda_{2}=\lambda_{3}=-1$, we have

$$
\frac{\eta}{s}=\frac{1.1 \times 10^{-5}}{4 \pi} .
$$

Here the constraints arising from $\langle\epsilon\rangle>0$ are satisfied.

Open Access. This article is distributed under the terms of the Creative Commons Attribution License (CC-BY 4.0), which permits any use, distribution and reproduction in any medium, provided the original author(s) and source are credited.

\section{References}

[1] D.T. Son and A.O. Starinets, Viscosity, Black Holes and Quantum Field Theory, Ann. Rev. Nucl. Part. Sci. 57 (2007) 95 [arXiv:0704.0240] [INSPIRE].

[2] S.A. Hartnoll, Lectures on holographic methods for condensed matter physics, Class. Quant. Grav. 26 (2009) 224002 [arXiv: 0903.3246] [INSPIRE].

[3] J. Casalderrey-Solana, H. Liu, D. Mateos, K. Rajagopal and U.A. Wiedemann, Gauge/String Duality, Hot QCD and Heavy Ion Collisions, arXiv:1101.0618 [INSPIRE].

[4] S. de Haro, S.N. Solodukhin and K. Skenderis, Holographic reconstruction of space-time and renormalization in the AdS/CFT correspondence, Commun. Math. Phys. 217 (2001) 595 [hep-th/0002230] [INSPIRE].

[5] K. Skenderis, Lecture notes on holographic renormalization, Class. Quant. Grav. 19 (2002) 5849 [hep-th/0209067] [INSPIRE]. 
[6] O. Hohm and E. Tonni, A boundary stress tensor for higher-derivative gravity in AdS and Lifshitz backgrounds, JHEP 04 (2010) 093 [arXiv:1001.3598] [INSPIRE].

[7] J. Smolic and M. Taylor, Higher derivative effects for 4d AdS gravity, JHEP 06 (2013) 096 [arXiv:1301.5205] [INSPIRE].

[8] N. Johansson, A. Naseh and T. Zojer, Holographic two-point functions for 4d log-gravity, JHEP 09 (2012) 114 [arXiv:1205.5804] [INSPIRE].

[9] S.-J. Hyun, W.-J. Jang, J.-H. Jeong and S.-H. Yi, Noncritical Einstein-Weyl Gravity and the AdS/CFT Correspondence, JHEP 01 (2012) 054 [arXiv:1111.1175] [INSPIRE].

[10] G. Giribet, A. Goya and M. Leston, Boundary stress tensor and asymptotically AdS3 non-Einstein spaces at the chiral point, Phys. Rev. D 84 (2011) 066003 [arXiv:1108.0400] [INSPIRE].

[11] Y. Kwon, S. Nam, J.-D. Park and S.-H. Yi, Holographic Renormalization and Stress Tensors in New Massive Gravity, JHEP 11 (2011) 029 [arXiv:1106.4609] [INSPIRE].

[12] E.A. Bergshoeff, O. Hohm, J. Rosseel and P.K. Townsend, Modes of Log Gravity, Phys. Rev. D 83 (2011) 104038 [arXiv: 1102.4091] [INSPIRE].

[13] G. Giribet and M. Leston, Boundary stress tensor and counterterms for weakened $A d S_{3}$ asymptotic in New Massive Gravity, JHEP 09 (2010) 070 [arXiv:1006.3349] [INSPIRE].

[14] M. Alishahiha and A. Naseh, Holographic renormalization of new massive gravity, Phys. Rev. D 82 (2010) 104043 [arXiv: 1005.1544] [INSPIRE].

[15] D. Allahbakhshi, M. Alishahiha and A. Naseh, Entanglement Thermodynamics, JHEP 08 (2013) 102 [arXiv: 1305.2728] [INSPIRE].

[16] S. Cremonini, J.T. Liu and P. Szepietowski, Higher Derivative Corrections to R-charged Black Holes: Boundary Counterterms and the Mass-Charge Relation, JHEP 03 (2010) 042 [arXiv:0910.5159] [INSPIRE].

[17] T. Faulkner, M. Guica, T. Hartman, R.C. Myers and M. Van Raamsdonk, Gravitation from Entanglement in Holographic CFTs, JHEP 03 (2014) 051 [arXiv: 1312.7856] [INSPIRE].

[18] J. Bhattacharya, M. Nozaki, T. Takayanagi and T. Ugajin, Thermodynamical Property of Entanglement Entropy for Excited States, Phys. Rev. Lett. 110 (2013) 091602 [arXiv: 1212.1164] [INSPIRE].

[19] D.D. Blanco, H. Casini, L.-Y. Hung and R.C. Myers, Relative Entropy and Holography, JHEP 08 (2013) 060 [arXiv: 1305.3182] [INSPIRE].

[20] V. Iyer and R.M. Wald, Some properties of Noether charge and a proposal for dynamical black hole entropy, Phys. Rev. D 50 (1994) 846 [gr-qc/9403028] [INSPIRE].

[21] R.-X. Miao, A Note on Holographic Weyl Anomaly and Entanglement Entropy, Class. Quant. Grav. 31 (2014) 065009 [arXiv: 1309.0211] [InSPIRE].

[22] A. Buchel, J. Escobedo, R.C. Myers, M.F. Paulos, A. Sinha et al., Holographic GB gravity in arbitrary dimensions, JHEP 03 (2010) 111 [arXiv:0911.4257] [INSPIRE].

[23] D.M. Hofman and J. Maldacena, Conformal collider physics: Energy and charge correlations, JHEP 05 (2008) 012 [arXiv: 0803.1467] [INSPIRE].

[24] D.M. Hofman, Higher Derivative Gravity, Causality and Positivity of Energy in a UV complete QFT, Nucl. Phys. B 823 (2009) 174 [arXiv:0907.1625] [InSPIRE]. 
[25] R.C. Myers, M.F. Paulos and A. Sinha, Holographic studies of quasi-topological gravity, JHEP 08 (2010) 035 [arXiv: 1004.2055] [INSPIRE].

[26] D.M. Hofman, Higher Derivative Gravity, Causality and Positivity of Energy in a UV complete QFT, Nucl. Phys. B $\mathbf{8 2 3}$ (2009) 174 [arXiv:0907.1625] [INSPIRE].

[27] H. Osborn and A.C. Petkou, Implications of conformal invariance in field theories for general dimensions, Annals Phys. 231 (1994) 311 [hep-th/9307010] [INSPIRE].

[28] J. Erdmenger and H. Osborn, Conserved currents and the energy momentum tensor in conformally invariant theories for general dimensions, Nucl. Phys. B 483 (1997) 431 [hep-th/9605009] [INSPIRE].

[29] R.C. Myers and B. Robinson, Black Holes in Quasi-topological Gravity, JHEP 08 (2010) 067 [arXiv: 1003.5357] [INSPIRE].

[30] A. Buchel, J.T. Liu and A.O. Starinets, Coupling constant dependence of the shear viscosity in $N=4$ supersymmetric Yang-Mills theory, Nucl. Phys. B 707 (2005) 56 [hep-th/0406264] [INSPIRE].

[31] R.C. Myers, M.F. Paulos and A. Sinha, Quantum corrections to eta/s, Phys. Rev. D 79 (2009) 041901 [arXiv:0806.2156] [INSPIRE].

[32] A. Buchel, R.C. Myers, M.F. Paulos and A. Sinha, Universal holographic hydrodynamics at finite coupling, Phys. Lett. B 669 (2008) 364 [arXiv:0808.1837] [INSPIRE].

[33] A. Buchel, R.C. Myers and A. Sinha, Beyond eta/s = 1/4 pi, JHEP 03 (2009) 084 [arXiv:0812.2521] [INSPIRE].

[34] A. Sinha and R.C. Myers, The Viscosity bound in string theory, Nucl. Phys. A 830 (2009) 295C-298C [arXiv: 0907 .4798] [INSPIRE].

[35] N. Banerjee and S. Dutta, Shear Viscosity to Entropy Density Ratio in Six Derivative Gravity, JHEP 07 (2009) 024 [arXiv:0903.3925] [INSPIRE].

[36] X.O. Camanho, J.D. Edelstein and M.F. Paulos, Lovelock theories, holography and the fate of the viscosity bound, JHEP 05 (2011) 127 [arXiv: 1010.1682] [INSPIRE].

[37] R. Brustein and A.J.M. Medved, Non-perturbative unitarity constraints on the ratio of shear viscosity to entropy density in UV complete theories with a gravity dual, Phys. Rev. D 84 (2011) 126005 [arXiv: 1108.5347] [INSPIRE].

[38] A. Buchel, J. Escobedo, R.C. Myers, M.F. Paulos, A. Sinha et al., Holographic GB gravity in arbitrary dimensions, JHEP 03 (2010) 111 [arXiv:0911.4257] [INSPIRE].

[39] R.C. Myers, M.F. Paulos and A. Sinha, Holographic Hydrodynamics with a Chemical Potential, JHEP 06 (2009) 006 [arXiv:0903.2834] [INSPIRE].

[40] S. Cremonini, K. Hanaki, J.T. Liu and P. Szepietowski, Higher derivative effects on eta/s at finite chemical potential, Phys. Rev. D 80 (2009) 025002 [arXiv:0903.3244] [INSPIRE].

[41] M. Brigante, H. Liu, R.C. Myers, S. Shenker and S. Yaida, Viscosity Bound Violation in Higher Derivative Gravity, Phys. Rev. D 77 (2008) 126006 [arXiv:0712.0805] [INSPIRE].

[42] M. Brigante, H. Liu, R.C. Myers, S. Shenker and S. Yaida, The Viscosity Bound and Causality Violation, Phys. Rev. Lett. 100 (2008) 191601 [arXiv:0802.3318] [INSPIRE].

[43] P. Kovtun, D.T. Son and A.O. Starinets, Viscosity in strongly interacting quantum field theories from black hole physics, Phys. Rev. Lett. 94 (2005) 111601 [hep-th/0405231] [INSPIRE]. 
[44] P. Kovtun, D.T. Son and A.O. Starinets, Holography and hydrodynamics: Diffusion on stretched horizons, JHEP 10 (2003) 064 [hep-th/0309213] [INSPIRE].

[45] G. Policastro, D.T. Son and A.O. Starinets, The Shear viscosity of strongly coupled $N=4$ supersymmetric Yang-Mills plasma, Phys. Rev. Lett. 87 (2001) 081601 [hep-th/0104066] [INSPIRE].

[46] L.-Y. Hung, R.C. Myers and M. Smolkin, On Holographic Entanglement Entropy and Higher Curvature Gravity, JHEP 04 (2011) 025 [arXiv:1101.5813] [INSPIRE].

[47] J. de Boer, M. Kulaxizi and A. Parnachev, Holographic Entanglement Entropy in Lovelock Gravities, JHEP 07 (2011) 109 [arXiv:1101.5781] [InSPIRE].

[48] A. Bhattacharyya, A. Kaviraj and A. Sinha, Entanglement entropy in higher derivative holography, JHEP 08 (2013) 012 [arXiv:1305.6694] [INSPIRE].

[49] D.V. Fursaev, A. Patrushev and S.N. Solodukhin, Distributional Geometry of Squashed Cones, Phys. Rev. D 88 (2013) 044054 [arXiv: 1306.4000] [InSPIRE].

[50] A. Bhattacharyya, M. Sharma and A. Sinha, On generalized gravitational entropy, squashed cones and holography, JHEP 01 (2014) 021 [arXiv: 1308.5748] [INSPIRE].

[51] X. Dong, Holographic Entanglement Entropy for General Higher Derivative Gravity, JHEP 01 (2014) 044 [arXiv: 1310.5713] [inSPIRE].

[52] J. Camps, Generalized entropy and higher derivative Gravity, JHEP 03 (2014) 070 [arXiv: 1310.6659] [INSPIRE].

[53] A. Bhattacharyya and M. Sharma, On entanglement entropy functionals in higher derivative gravity theories, arXiv:1405.3511 [INSPIRE].

[54] M. Henningson and K. Skenderis, The Holographic Weyl anomaly, JHEP 07 (1998) 023 [hep-th/9806087] [INSPIRE].

[55] S. 'i Nojiri and S.D. Odintsov, On the conformal anomaly from higher derivative gravity in AdS/CFT correspondence, Int. J. Mod. Phys. A 15 (2000) 413 [hep-th/9903033] [inSPIRE].

[56] K. Sen, A. Sinha and N.V. Suryanarayana, Counterterms, critical gravity and holography, Phys. Rev. D 85 (2012) 124017 [arXiv:1201.1288] [INSPIRE].

[57] C. Imbimbo, A. Schwimmer, S. Theisen and S. Yankielowicz, Diffeomorphisms and holographic anomalies, Class. Quant. Grav. 17 (2000) 1129 [hep-th/9910267] [INSPIRE].

[58] S. Deser and A. Schwimmer, Geometric classification of conformal anomalies in arbitrary dimensions, Phys. Lett. B 309 (1993) 279 [hep-th/9302047] [InSPIRE].

[59] F. Bastianelli, S. Frolov and A.A. Tseytlin, Conformal anomaly of (2,0) tensor multiplet in six-dimensions and AdS/CFT correspondence, JHEP 02 (2000) 013 [hep-th/0001041] [INSPIRE].

[60] S. Deser, H. Liu, H. Lü, C.N. Pope, T.C. Sisman et al., Critical Points of D-Dimensional Extended Gravities, Phys. Rev. D 83 (2011) 061502 [arXiv:1101.4009] [INSPIRE].

[61] H. Liu and A.A. Tseytlin, $D=4$ super Yang-Mills, $D=5$ gauged supergravity and $D=4$ conformal supergravity, Nucl. Phys. B 533 (1998) 88 [hep-th/9804083] [INSPIRE].

[62] X.O. Camanho, J.D. Edelstein and J.M. Sánchez De Santos, Lovelock theory and the AdS/CFT correspondence, Gen. Rel. Grav. 46 (2014) 1637 [arXiv:1309.6483] [INSPIRE].

[63] J.D. Edelstein, Lovelock theory, black holes and holography, arXiv:1303.6213 [INSPIRE]. 
[64] X.O. Camanho and J.D. Edelstein, Causality in AdS/CFT and Lovelock theory, JHEP 06 (2010) 099 [arXiv: 0912.1944] [INSPIRE].

[65] X.O. Camanho and J.D. Edelstein, Causality constraints in AdS/CFT from conformal collider physics and Gauss-Bonnet gravity, JHEP 04 (2010) 007 [arXiv:0911.3160] [INSPIRE].

[66] G.T. Horowitz and N. Itzhaki, Black holes, shock waves and causality in the AdS/CFT correspondence, JHEP 02 (1999) 010 [hep-th/9901012] [INSPIRE].

[67] J. de Boer, M. Kulaxizi and A. Parnachev, Holographic Lovelock Gravities and Black Holes, JHEP 06 (2010) 008 [arXiv: 0912.1877] [INSPIRE].

[68] J. de Boer, M. Kulaxizi and A. Parnachev, $A d S_{7} / C F T_{6}$, Gauss-Bonnet Gravity and Viscosity Bound, JHEP 03 (2010) 087 [arXiv:0910.5347] [INSPIRE].

[69] M.F. Paulos, Transport coefficients, membrane couplings and universality at extremality, JHEP 02 (2010) 067 [arXiv: 0910.4602] [INSPIRE].

[70] N. Banerjee and S. Dutta, Near-Horizon Analysis of eta/s, Nucl. Phys. B 845 (2011) 165 [arXiv:0911.0557] [INSPIRE].

[71] J.L. Cardy, Is There a c Theorem in Four-Dimensions?, Phys. Lett. B 215 (1988) 749 [INSPIRE].

[72] Z. Komargodski and A. Schwimmer, On Renormalization Group Flows in Four Dimensions, JHEP 12 (2011) 099 [arXiv: 1107.3987] [INSPIRE].

[73] L. Girardello, M. Petrini, M. Porrati and A. Zaffaroni, Novel local CFT and exact results on perturbations of $N=4$ super Yang-Mills from AdS dynamics, JHEP 12 (1998) 022 [hep-th/9810126] [INSPIRE].

[74] D.Z. Freedman, S.S. Gubser, K. Pilch and N.P. Warner, Renormalization group flows from holography supersymmetry and a c theorem, Adv. Theor. Math. Phys. 3 (1999) 363 [hep-th/9904017] [INSPIRE].

[75] R.C. Myers and A. Sinha, Seeing a c-theorem with holography, Phys. Rev. D 82 (2010) 046006 [arXiv: 1006.1263] [INSPIRE].

[76] R.C. Myers and A. Sinha, Holographic c-theorems in arbitrary dimensions, JHEP 01 (2011) 125 [arXiv: 1011.5819] [INSPIRE].

[77] A. Sinha, On higher derivative gravity, c-theorems and cosmology, Class. Quant. Grav. 28 (2011) 085002 [arXiv: 1008.4315] [INSPIRE].

[78] J.T. Liu, W. Sabra and Z. Zhao, Holographic c-theorems and higher derivative gravity, Phys. Rev. D 85 (2012) 126004 [arXiv: 1012.3382] [INSPIRE].

[79] Y. Nakayama, Does anomalous violation of null energy condition invalidate holographic c-theorem?, Phys. Lett. B 720 (2013) 265 [arXiv:1211.4628] [InSPIRE].

[80] A. Bhattacharyya, L.-Y. Hung, K. Sen and A. Sinha, On c-theorems in arbitrary dimensions, Phys. Rev. D 86 (2012) 106006 [arXiv:1207.2333] [INSPIRE].

[81] M.F. Paulos, Holographic phase space: c-functions and black holes as renormalization group flows, JHEP 05 (2011) 043 [arXiv:1101.5993] [INSPIRE].

[82] C.A. Agon, M. Headrick, D.L. Jafferis and S. Kasko, Disk entanglement entropy for a Maxwell field, Phys. Rev. D 89 (2014) 025018 [arXiv:1310.4886] [INSPIRE]. 
[83] S. Raju, Four Point Functions of the Stress Tensor and Conserved Currents in $A d S_{4} / C F T_{3}$, Phys. Rev. D 85 (2012) 126008 [arXiv:1201.6452] [INSPIRE].

[84] A. Dymarsky, On the four-point function of the stress-energy tensors in a CFT, arXiv:1311.4546 [INSPIRE].

[85] D. Grumiller and O. Hohm, $A d S_{3} / L C F T_{2}$ : Correlators in New Massive Gravity, Phys. Lett. B 686 (2010) 264 [arXiv:0911.4274] [INSPIRE].

[86] D. Grumiller and I. Sachs, $A d S_{3} / L C F T_{2}$ - Correlators in Cosmological Topologically Massive Gravity, JHEP 03 (2010) 012 [arXiv:0910.5241] [INSPIRE].

[87] D. Grumiller, M. Irakleidou, I. Lovrekovic and R. McNees, Conformal gravity holography in four dimensions, Phys. Rev. Lett. 112 (2014) 111102 [arXiv: 1310.0819] [INSPIRE].

[88] S. Banerjee, A. Bhattacharyya, A. Kaviraj, K. Sen and A. Sinha, Constraining gravity using entanglement in AdS/CFT, JHEP 05 (2014) 029 [arXiv:1401.5089] [INSPIRE]. 